Oregon Coast

buds. 5563 \& 5611

May \& \& May 29, 1914 
507 
Oragon Coxst $x<$

Tay 8-

Nydro. $5563-5611$ 
Bydo 5563 to 5611

May 8 t may29,1914 


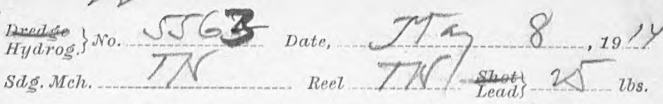

Turns 164 .... Corn Depth 89 fms.

Sag. Cup Arming Amt. Bot'm

Character of hod 8

Thermos. $\left\{\begin{array}{l}\text { Make } \\ \text { No. }\end{array}\right.$

$$
\text { Corn }\left\{\begin{array}{l}
\text { Instr. } \\
\text { Stem. }
\end{array}\right.
$$

Bottom Reading Cor'd Temp.

Temp. Surf. $\left\{\begin{array}{l}5 \% \\ \end{array}\right.$

Time $5.34,5, \quad 54^{4}$

Time

Density: Surf. fins.

Wind: Direction SE

Force $3-4$ Baronet. $29 \cdot 92$ Ant of 10 State of
Sea.

Weather $\%$

Direction and Force
of Current. $\left\{\begin{array}{l}\text { Surface. } \\ \text { Bottom. }\end{array}\right.$

Tide

General Locality Off Oregon Cos est Positiontillamookfiyluh bears $866^{\circ} \mathrm{E}$ (thu) distant
28.8 milled

Chart used: No

6100 $E d ' n$

Drift: Direction Distance.

Depth of Haul

Apparatus and Rig used

Time towing h. $m$.

Remarks:

Deck offer Mr. Stick.

Recorder

Aryllit:- 
PM. Down. Er. PM. FMS. $5345 \% 544$

… M. Down. UP. …M.

1900

2000

2100

2200

2300

2400

2500

2600

2700

2800

2900

3000

3100

3200

8300

8400

3500

3600

3700

3800

3900

4000

4100

4200

4300

4400

4500

4600

4700

4800 
23. Dredge
Hydro
Sag.Mch. Turns 12) Corn Depth . Q5 fms. Sis. Cup Arateing Amt. Bot'm Character of

Hols

Thermom. $\left\{\begin{array}{l}\text { Make } \\ \text { No. }\end{array}\right.$
Bottom Reading Corn $\left\{\begin{array}{l}\text { Instr. } \\ \text { Stem. }\end{array}\right.$ Cor'd Temp.

Temp. Surf. \{ $\leqslant 20$

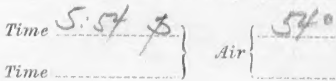
Density: Surf. fms.

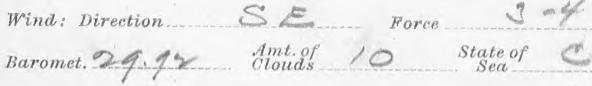
Weather 0

Direction and Force
of Current. $\left\{\begin{array}{l}\text { Surface } \\ \text { Bottom }\end{array}\right.$

Tide.

General Locality Of Oregon Cory Position Tillamookfight hears $864^{\circ} \mathrm{F}(\mathrm{tm})$ distant
29.3 miles chart used: No. 6100 $E d ' n$ Drift: Direction Distance.

Depth of Haul.

Apparatus and Rig used

Time towing h.

Remarks:

Tine

bios F.M. monet well Ax y hols

8:30 A.M. (may q) $\delta-3.0$ 54.0 53.0 $5-3.8$

Deck of feer Mr. Click Recorder... $=\left(72 \frac{71 \%}{11-1023}\right.$ 
SOUNDING WIRE.

$P$ M. Down. UP. $\perp$ M. $554: 558$
TREDGING CABLE.

100

200

300

400

500

600

700

800

900

1000

1100

1200

1300

1400

1500

1600

1700

1800

1900

2000

2100

2200

2300

2400

2500

2600

2700

2800

2900

3000

3100

3200

3300

3400

3500

3600

3700

3800

3900

4000

4100

4200

4800

4400

4500

4600

4700

4800

4900
..... M. Down. UP. ..... M.

\begin{tabular}{l|l|l|}
\hline+1 & \\
\hline
\end{tabular}

-

0


I

Druse 1 Ho. 556 Date,

$S d g . M c h$.

Reel Shot $2 \mathrm{v}^{-} . \mathrm{us}$.

Turns... So 3 Corn Depth $2 \%$ fins.

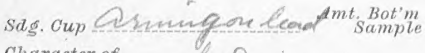

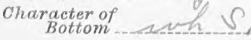

Thermom. $\left\{\begin{array}{l}\text { Make .......... } \\ \text { No. }\end{array}\right.$ Corn $\begin{array}{l}\text { Instr. } \\ \text { Stem. }\end{array}$

Bottom Reading Cor'd Temp.

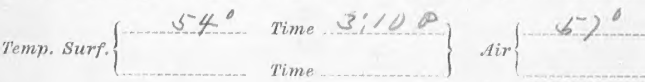

Density: Surf. fins.

Wind: Direction

Force

2

Baronet. $30.0 s^{-}$Amt of 6

State of

Weather ...

Direction and Force
of Current. $\left\{\begin{array}{l}\text { Surface } \\ \text { Bottom }\end{array}\right.$

Tide

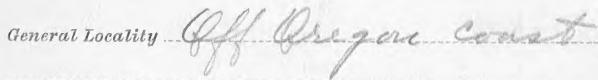
Position Yaquima Right hears $123 \%$ Eta) distant chart used: No 6000 Eden

Drift: Direction Distance

Depth of Haul

Apparatus and Rig used

Time towing $h$. $m$.

Remarks :

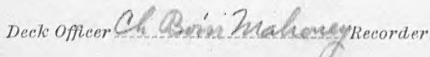

$11+1023$ 
FMS.

DREDGING CABLE.

31062000700

0

(

M. Down. UP. … M.

100

200

300

400

500

600

700

800

900

1000

1100

1200

1800

1400

1500

1600

1700

1800

1900

2000

2100

2200

2300

2400

2500

2600

2700

2800

2900

3000

3100

3200

3300

3400

3500

3600

3700

3800

3900

4000

4100

4200

4300

4400

4500

4600

4700

4800

4900
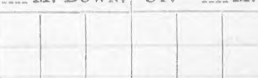

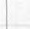

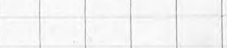




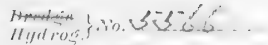

nate,

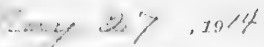

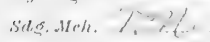

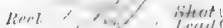

lls.

I'urns $\quad$ Cor'n

l)epth

fims

Silot (iul) Amt Rot'm

Character of

Hollom 2 on

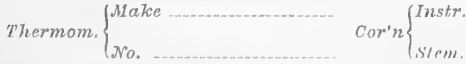

Boltom Reatins

Cior't T'rmp.

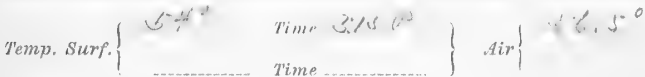

Density: Surf.

fms.

Wind: Direction QP $x$,

Toree 2

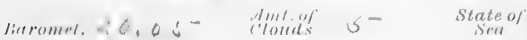

Weather sere

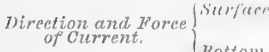

Till

General Locatity

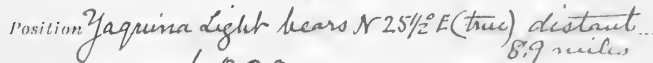
chart usert: No, 6000 Trl'n

Drift: Difretion Distonter

Depth of 11 aul

Amparatus and lig used

limmork:s 
SOUNDING WIRE.

Q M. Down. UP. $\rightleftharpoons$ M. $3 / 6|30| 3 \mid 1860$
FMS.

DRTADGING ('AISLE:

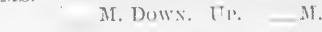

200

3300

100

500

(it)

700)

800

(9)11

1000

1100

$1: 200$

1300

1.100

1500

$1(600)$

1700

1800

1900

2000

$\because 100$

2500

$2: 300$

$\therefore 100$

2501

26100

2700

2800

2900

$: 3000$

$\$ 3100$

$: 3 \div 00$

:3:300

$: 1(*)$

3500

3600

:

3400

$\$ 900$

4000

4100

(1200

4:301)

.1800

4500

$4(6) 0$

.1700

4800

(4900) 


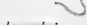

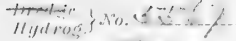

1)ate, ( $\ldots, \ldots, 0)$

, 10) 4

sils. Hethe /

Repl

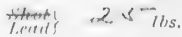

Turns $\leq+6$

$\operatorname{Cor}^{\prime} n$

Depth: 30

fms.

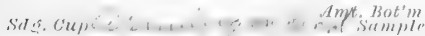

Character of
Botlom

thermom. $\left\{\begin{array}{l}\text { Make } \\ \text { Mi). }\end{array}\right.$

Botlom Rerulins

Temp. Surf. $\left\{\begin{array}{c}-40 \\ \ldots\end{array}\right.$

Density: Surf.

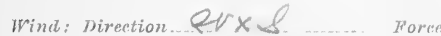

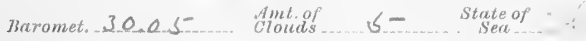

Weather

Direction and Force
of Current.

Tide

Ginerel Lomelit!

Cor'n $\left\{\begin{array}{l}\text { Instr. } \\ \text { stem. }\end{array}\right.$

('or'd Trm)

Time 3is.a.P. . Air $\}$ is 10

fims.
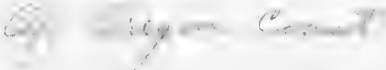

"osition Yaquina Lyght hears $1255_{2}^{\circ}$ E(thw) distunt.

chart userl: No. 6000

$T d^{\prime} n$

1)rift: Direction

Distance

Jepth of Haut

Apparatus and Ris used.

limuerlas: 
$11-102: 3$

\begin{tabular}{l|l}
\hline SOUNDING WIRF. & DREDGING CABLE.
\end{tabular}

a M. Down. Ur. $\infty$ M. T. T. Down. UP. … M. $3 \div 6 ; 3 \div 02 \quad 11$

100

$2(1)$

:300

100

i)( ()

(i) ()

700

신)

(9)10)

1000

1104

1200

1300

1.400

1500

1600

1700

1800

1900

2000

2100

2004

$2: 300$

$\because 100$

s)

$\because$ (i)

ะร)

?SA(0)

$\because 90(0)$

:3001)

3100

:2:(1)

:3:it)

:31(1)

涪(0)

:3(100)

:ห⿰ด)

:खsto

$\sin (0)$

.10006

1100

f. $\div(1)$

1:300

1100

1500

$f(0) 0$

17)(

arital

1900 


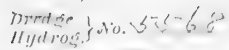

I) Ut',

$$
1
$$
$19>1$

Sug. Mch. . Y Reet

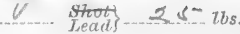

Turns s $6-8$

$\operatorname{Cor}^{\prime} n$ fms.

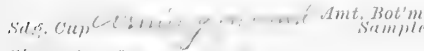

(Meractera) liollom

Thermom. $\left\{\begin{array}{l}\text { Mak } \\ \text { No }\end{array}\right.$ Cor'n $\left\{\begin{array}{l}\text { Instr. } \\ \text { Stem. }\end{array}\right.$

liotlom Reralins

corid Trmp.

Temp. Surf. $\left.\left\{\begin{array}{ll}-344^{\circ} & \text { Time } 3: 400 \\ \text { Time }\end{array}\right\} \operatorname{Air}\right\}$

Jensity: Surf.

fins.

Wind: Direction. Force ..... 2

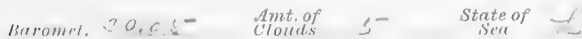

Weather ... Wres.

7)irection and Force
of Current.

Tide

Generat Lomelity
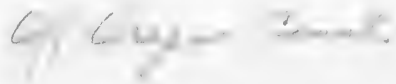

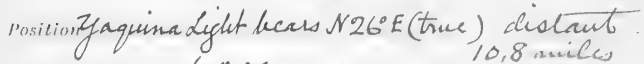
chart used: No.

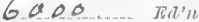

Hrift: Dirertion

Distertert

Denth of Harl

Apparatus and Ris used

Time towing .

h.

ili.

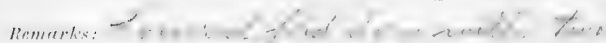

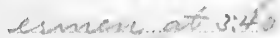

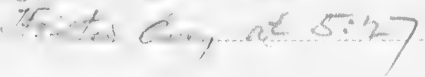


$11-1023$

SOUNDING WHRE.

JI. Down' UI. en.

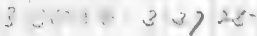

DRIODTING ('ABLLE.

FMIS.

MT, Dow. (T1. .. M.

10

100

200

:3)(1)

400

.00

(i) $(0)$

7ก)

Si(k)

(10)

1000

1100

12(1)

$1301)$

1400

1500

1600

1700

$1+200$

1900

2000

2100

2200

2300

2100

200

$2(6)$

$2 \pi 0()$

खर

:ख)

¿3000

$\$ 100$

$: \because \cdot 0)$

:3:300)

:314

initio

:31000

:3ั)

:350)(1)

:89)

1000

1100)

12001)

f:s(t)

$14(01)$

1,000

\&(io)

ticou

4800

4000 
Itrand sts

III diong

Jute,

$\pm 2 \quad, 70 \%$

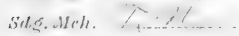

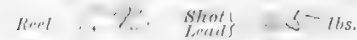

Turns $\quad$.. Cor

Depth .... 34 fms.

Salo, orup

Amt. Trot'm

Character of Boltomi

Thermom. $\left\{\begin{array}{l}\text { Mak } \\ \text { No. }\end{array}\right.$ ior'n $\left\{\begin{array}{l}\text { lustr. } \\ \text { stem. }\end{array}\right.$

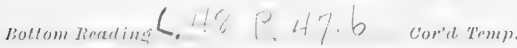

Timp. Surf. $\left\{\begin{array}{ll}.5 & \text { Time }: 1, \\ & \text { Time }\end{array}\right\}$ Airo

I)ersit!: Surf:

fms.

Inimd: llirention

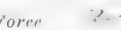

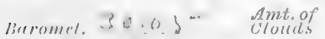

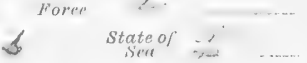

Weatlerr , $C$

Direction and Force
of Gurrent. $\left\{\begin{array}{l}\text { Sur/ace } \\ \text { Bullom }\end{array}\right.$

Tide

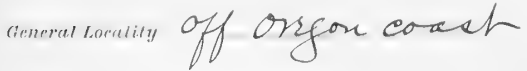

'ositionYaquina Light heass $N 27^{\circ}$ E(true) divtant ... Chart used: No, 6000 . Fan'n

Hrifl: Hisertion

Distance.

Depth of Haul

Apparatus and litg used

34

Tim) Iomints

h.

$m$.

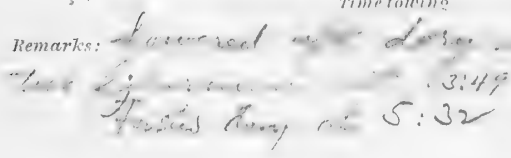


SOUNDING WIRE.

II. TOWN. TH, M.

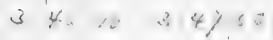
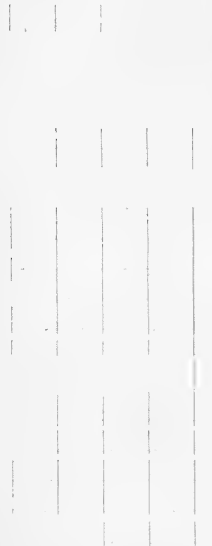

FMS.

DIELGING CAHEH:-
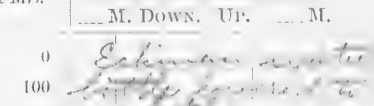

200

300 3 5100 if 6 : :0

I(K)

$3 \mathrm{~s} s$

500

(io)

700

800 \& $\because \because \frac{1}{-1}+2$

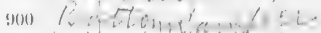

$1000 \cdot 4 \cdot 04-4 / 0520$

$1100: 40420 \backsim$ o 30

1200

1800 Mosemple

1400

1500)

1600

1700

1800

1000

2000

2100

2200

2300

2400

2500

2600

2700

2800

2900

3000

3100

3200

3300

3400

3500

3000

3700

$: 3800$

3000

4000

4100

4200

4300

4400

4500

4600

4700

4800

4900 
6

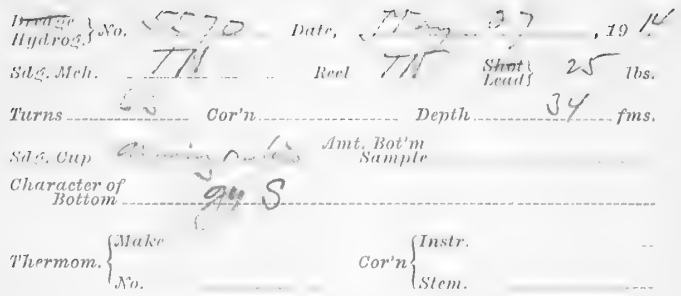

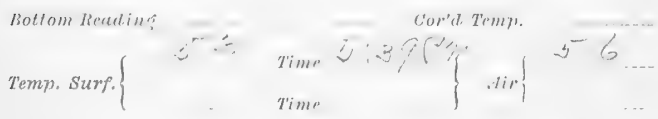

Density: Surf.

Wind: Direction.

Burrow. $30,3 \mathrm{~S}^{-}$Ant of

Force

fms.

State of
Set

Weather LC

Direction and Force
of Current.

Tide

annoral Locality OE C

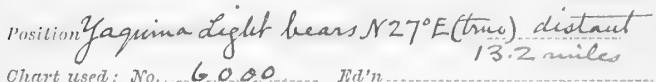
Chant used: No. 6000 Win.

Drift: Direction Distance

Depth of Hath

Apparatus and Rig used.

Time lowing

h. $m$.

ficmertis:

Deckoplere 
SOUNDING WIIRE.

T. DOWN. UI. O M. $5 \sqrt{38} 30$ 5 40 wo
I)REDTING CABLAL.

FMS. 1.... MT. Dows. U1. _ _. M.

100

200

;i) (1)

14)

500

(i) ()

־0()

S(1)

(3)( )

1000

110()

$12(1)$

$13(0)$

1.100

1500

1600

1700

1800

1900)

)(1)()

2100

2000

2):30

3100

2500

$\because 60)$

$2 \%(0)$

$25(0)$

:)(1)(0)

$: 30(10)$

3100

$: 3 \div 0(x)$

:31:00

:31010)

:3\%(1)

$: 3000$

:30 (1)

m(i)

:290)

. I 1$)(1)$

+1 $1(x)$

1200

1:300

.100

. 1500

$4(10)$

4700

1800

1900 


\section{SOLINJING IVIRE.}

I... M. Down. Um. $\left.\right|_{\text {.... M. }}$ $606 \quad 67300$

FMS.

DRFDGING CAIBI,E.

|... M. Down.| UP. _M.

$2(\xi)$

$: 3())$

.100

500

(i) (r)

\%0)(

$\mathrm{sinO}$

(1)()

1000

1100

1:())

$1300)$

1.100

1500

1600

1700

1800

1900

2000

$\because 100$

2000

(2) $(1)()$

$\therefore 100$

:50

2600

$\because(k)$

:2400

o(3)()

:000

$\$ 100)$

$\because: 300$

:3is()0)

:i100

3is)(1)

:360)

:3\%(0)

:300

:1900

.1000

.1100

1200

$1: 200$

4.100

150

s(60)

$470(1)$

1800

490)

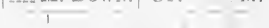


8

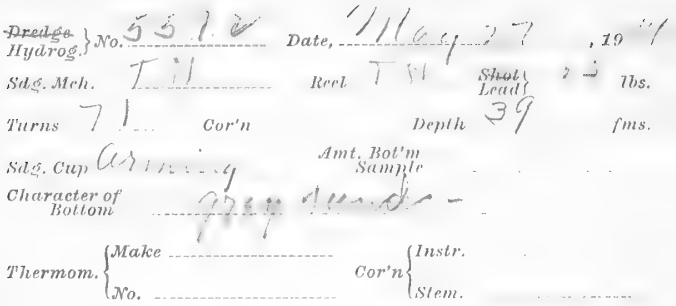

Bottom Reading Cor'd Temp.

Temp. Surf. $\left.\left\{\begin{array}{ll}\text { Time } & \text { Time }\end{array}\right\}, \operatorname{lir}\right\}$

Density: Surf.

rims.

Wind: Direction

Fore e

Iiaromet. 30.3 5 Amt of . 2 State of

Weather

tc

Direction and Force
of Current. $\left\{\begin{array}{l}\text { Surface } \\ \text { Bottom }\end{array}\right.$

Tide.

General. Loeatily

position Jaquina Light hears $31^{\circ} \mathrm{E}$ (true) distant

chert herl: wi. 6000

Hit'

Drift: Direction

Distance

Depth of Haul

Apparatus and Ri@ ned.

Remerrls:

Time touring t

$m$.

Derek. Offerer

Recorder

h. 
$\frac{11-1023}{\text { SOUNDINC+ WIRT. }}$

P. M. Down: Ur. P M. $6|31-6| 33-$
FMS.

DREDGING CABLE.

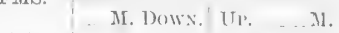

100

200

$: 300$

.100

500

(60)

700

800

(900)

1000

1100

1200

1300

1400

1500

1000

$1 \% 00$

1800

1900

2000

2100

2200

2300

2400

2500

2600

ฉ\%(0)

2900

290010

$: 000$

3100

:3200

: 300

:3100

3500

$: 3600$

$: 3700$

$\$ 800$

$: 9900$

4000

$\$ 100$

4200

4300

4.100

4500

4000

$+1700$

4800

4900

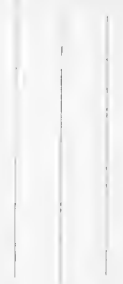




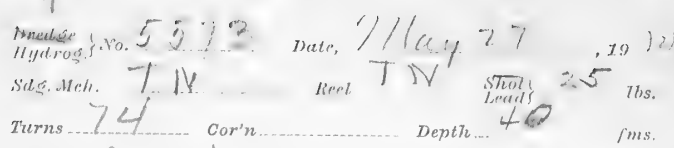
Sag. Cup Curmminy - Amt. Bot'm

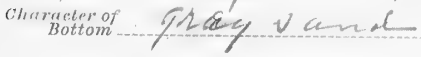

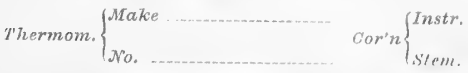

Buttom Realins

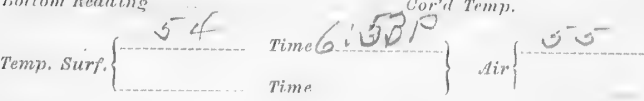

Densily: Surf:

fims

Wind: Direction.207 Foree 3

Haromet. $30: 35$ Amt of 2 State of 1

Ihealtier

7) irection and Foree
of Current. $\left\{\begin{array}{l}\text { Surface } \\ \text { Bottom }\end{array}\right.$

Tide

(innmal Lorretily

PositionYaquma Light hears $\$ 30^{\circ} \mathrm{E}$ (true) destrut chart used: No. 6000 It't'n

Mrift: Dirention

Distene

Depth of Haul

Apparatus and Rig used

limmarlis: 
SOUNDING WIRE.

$P$ m.Down. U1. F....

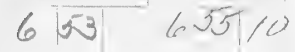

DREDGING CABLE.

FASS.

M. DOW. UP. M. M.

1)

100

200

$: 300$

400

500

(600)

700

800

(90)

1000

1100

1200

1300

1400

1500

1600

1700

1800

1900

2000

2100

2200

2300

2400

2500

2600

2700

2800

2900

$: 3000$

$\$ 100$

3200

:300

3100

$3 \bar{i} 00$

$\$ 600$

$: 3700$

$\therefore 800$

3900

1000

4100

4200

4300

4100

4500

1600

$1 \% 00$

1800

.1900 


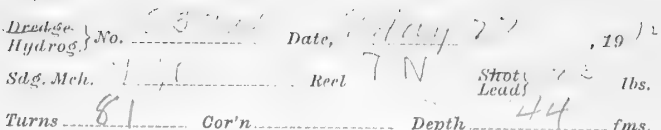

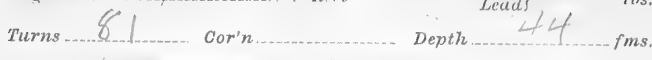

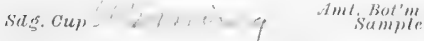

Character of
Bottom

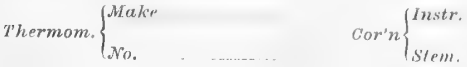

Bottom Reading

Cor'd Temp,

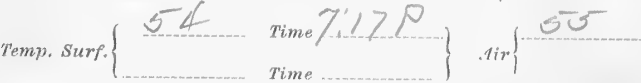

Density: Surf

Wind: Direction: 2

fims.

Find: force

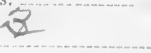

Buromet. $30 . \overline{3} \quad \begin{gathered}\text { Amt.or } \\ \text { Clotids }\end{gathered}$

Weather

c C

Direction and Force
of Current.

Tide

General Locality?

Positionfieceta Light heas $\$ 45^{\circ}$ E (true) distriot.

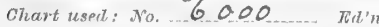

Drift: Direction

Distance.

Depth of Haul

Apparatus and Ri@ used

Remurh:s:

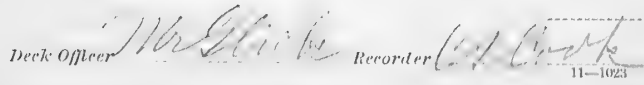


SOUNDING TVIRE.

M. Down. U1?. MI.

$7117+719$
DRFDGING $\div$ CABLE.

FMS

0

100

200

300

400

500

600

700

800

900

1000

1100

1200

1800

1400

1500

1600

1700

1800

1900

2000

2100

2200

2300

2400

2500

2600

2700

2800

2900

3000

3100

8200

3300

3400

3500

3600

3700

3800

3900

4000

4100

4200

4300

4400

4500

4600

4700

4800

4900

(... M. Dows. Ur. ... M.<smiles>C1CCC(C2CCCC2)C1</smiles> 
Turns /f?

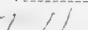

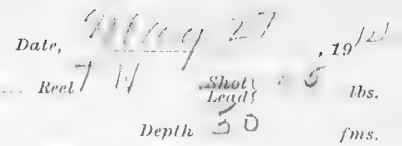

Sals. $C u$ j)

character of Bottom

T'hermom. $\left\{\begin{array}{l}\text { Make } \ldots o r n \\ \text { No. }\end{array}\left\{\begin{array}{l}\text { Instr. } \\ \text { Stem. }\end{array}\right.\right.$

Bottom Reading .

Temp. Surf. \{

Cor'n Amt. Bot'm
Simile

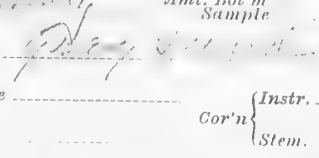

Density: Surf.

Wind: Dirertion Cor'd Temp.
Baromet. $36.3-$ Mmloof

Weather BC

Time $\%(1)$

Time

$\operatorname{Air}$

fims.

Direction and Force
of Current. $\left\{\begin{array}{l}\text { Surface } \\ \text { Bottom }\end{array}\right.$

Tide

Generat Loealily

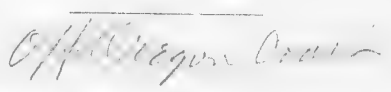

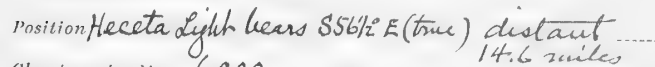
chrort usert: Ao. 6000

Eal'n.

Drift: Direstion

Distance

Depth of Haul

Apparatus and Ries used.

Time towing

$h$. ni.

\section{Remurlis:}

\section{Time}

8:03 F.M.
Not bulk Dy null

48.5
$6-3.0$ 

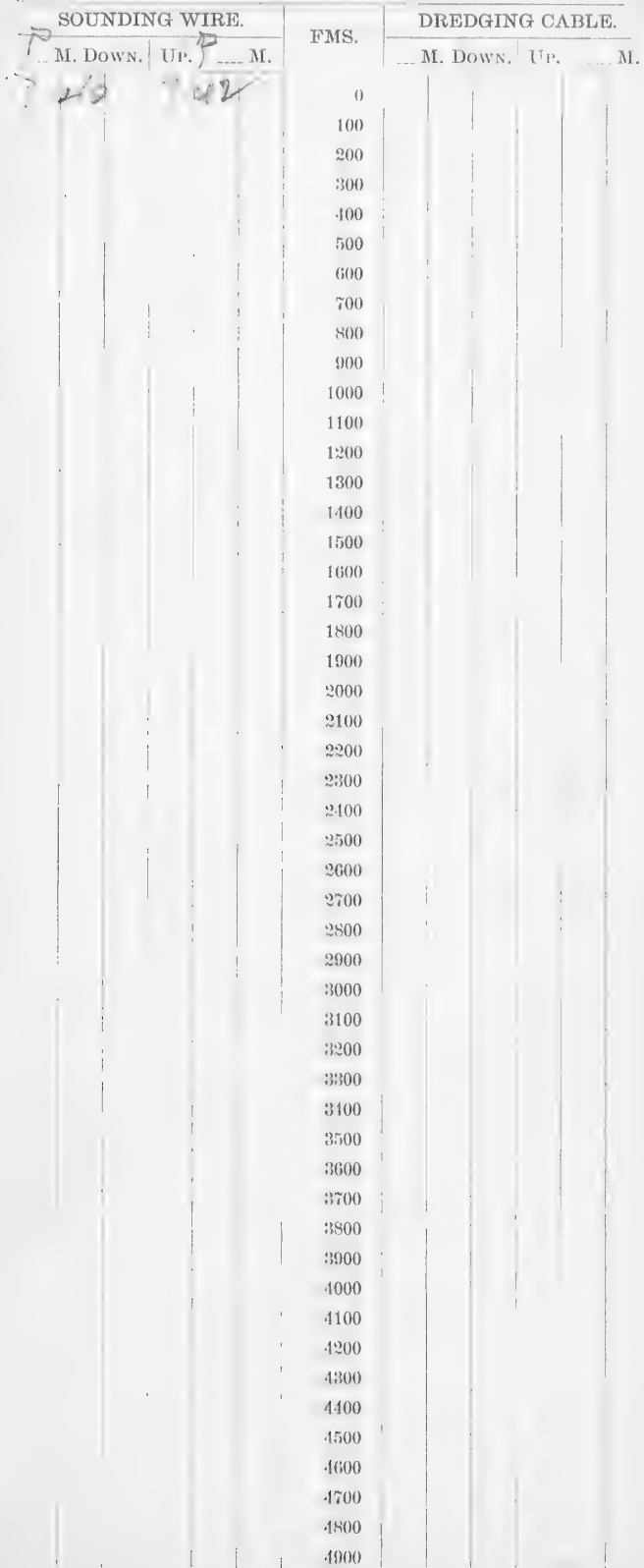
T) SOUNDING WIRE.

1) M. Down. UP. T M. 8103.3018106
FMS.

DREDAINA (ABLE.

0

100

200

$\$ 300$

400

500

(i) 0

ז00

$\mathrm{SO0}$

()) $(0)$

1000

110)

1200

1300

1400

1500

1600

1700

1800

1900

2000

2100

$2 * 200$

$2: 30()$

2100

2500

2600

2700

2500

2000

3000

3100

3200

3300

3100

3500

8600

$\$ 700$

$\$ 8800$

13900

4000

4100

$4: 200$

$4: 300$

4400

4500

4600

1700

4800

4900

M. Down.| UP. …...

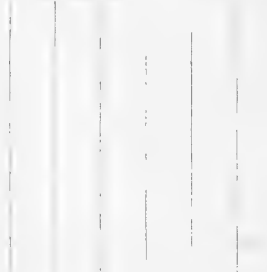




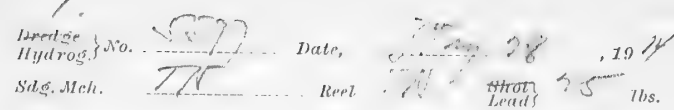

$$
\begin{aligned}
& \text { nepth } 53 \text { fins. }
\end{aligned}
$$

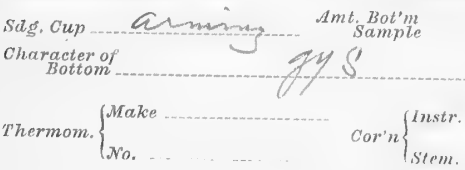

Bottom Reading.

Cor'd Temp.

Temp. Surf. $\left\{\begin{array}{ll}\text { Time } & \text { Time }\end{array}\right\}$. Tir $\}$

fims.

Nensity: Surf.

Wind: Direction...

Buromet. $30.4 / 2$ Ant.of

Weather

ac

Direction and Force
of Current. $\left\{\begin{array}{l}\text { Surface } \\ \text { Bottom }\end{array}\right.$

Tide

General Locality ....

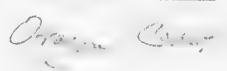
"osition Heceta Light hears $S 79 / 2 E($ thin) dictant

chart used: No, 6OOO ... Ed'n

Drift: Direction

Distance

Depth of Hauz

Apparalus and Rig used

Time towins

h.

m.

liemarks: 
SOUNDING TVIRT.

A M. DOWn. Ur. Z M.

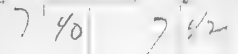

FMS.

DREDGING CABLE.

II. Down. U1. … MI.

1)

100

200

300

11)0

500

(i) 00

700

800

()00

1000

1100)

1200

1300

1400

1500

1600

1700

1800

1900

2000

2100

200

$2: 300$

2400

2500

2600

$2 \% 00$

2800

2900

$: 3000$

$\$ 3100$

3900

33300

3100

3500

$: 3600$

$3 \%$

3800

$3: 3900$

4000

1100

4200

1:3010)

4400

4500

4600

4700

4800

4900 
Hormtiase

IIyliogs fio.

Dettr,

Sal. $M \circ$.

Inepl

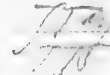

thot

912

Turns

Depth

61

Sal. Cup olumann Ami. Bot'm

Character of Bottom $\infty_{x+2}$

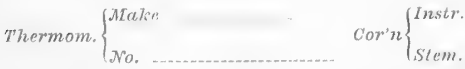

Bottom Reading

Cor'd Temp.

Temp. Surf.

Timp $8: \dot{0}$ ? $\{$ ar $\}$

Jensity: surf:

fins.

Wind: Direction

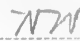

3

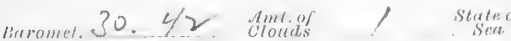
fms.

weather the

Direction and Worce
of Current.

Wider

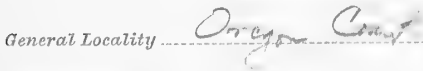

Posilion Heceta Light hears $88^{\circ}$ E (true) dielaut ohart used: No $6000 \ldots . .$. Fin.

Mrifl: Direrfion

Disfaner

Depth of Haul

Apparatus and Rig used.

liemurles:

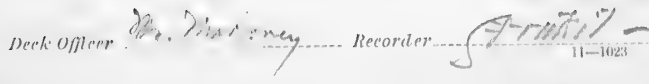


$11-1023$

SOUNDING WIRE. $\Leftrightarrow$ M. Down. U⿴囗十 $\mathrm{U}_{1}$. 8092
FMS.

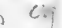

DREDGING CABLE.

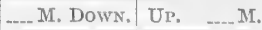

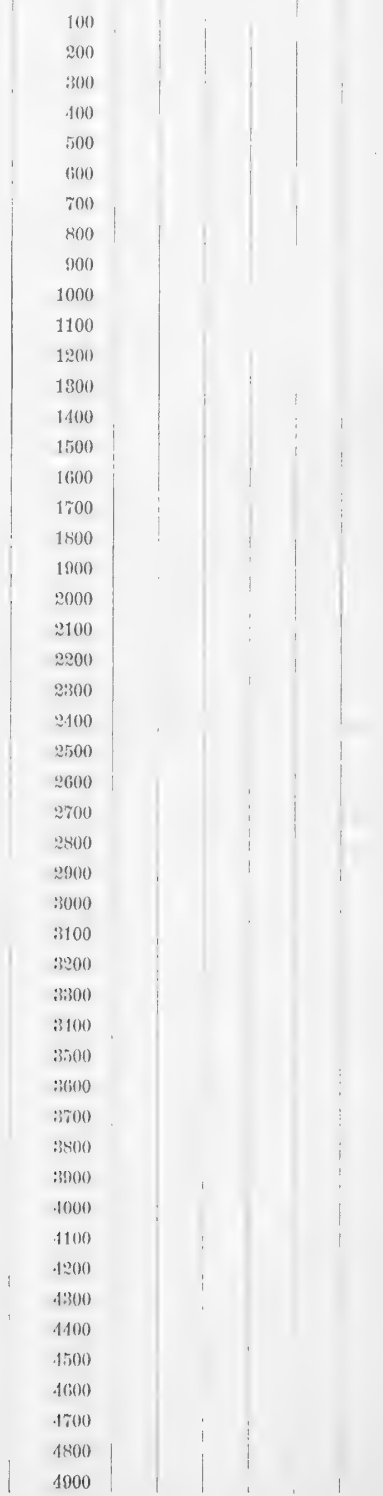




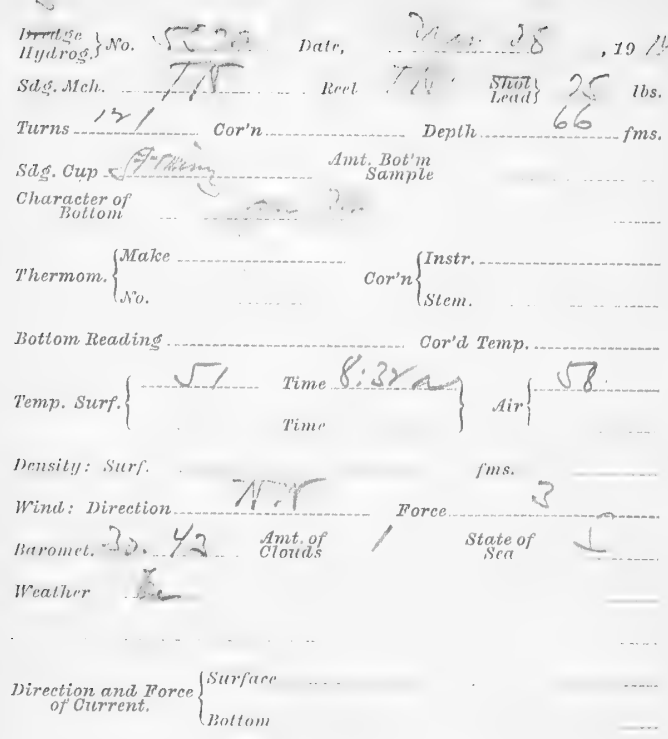

Tille

Goneral locatity

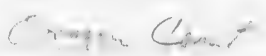

(

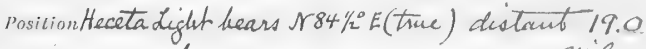 ohart used: vo 6000 Nin miles.

Drift: Direetion

Distance.

Depth of Haul

Apparatus and Rig used

Remrerk:

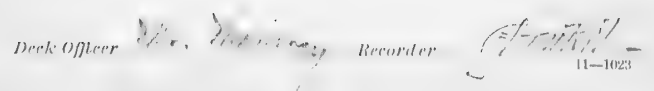


SOUNDINA WIIR]:

fT M. Down.| Ur. F? M.

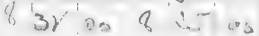

DREDFING (:ATLIX

FMS.

M. Down. Ur. …. M.

1)

10)

200

:300

100

500

(6)0

700

solo

900

1000

1100

1200

1800

1400

1500

1600

1700

1800

1900

2000

2100

2200

2300

$\approx 100$

2500

2600

ชัน

250

2900

$: 3000$

$\$ 100$

$: 3200$

:3*00)

3900

3)

$\because 6000$

3\%

3800

:3900

to00

.1100

1200

4300

4100)

4500

f600)

4700

4800

4900 

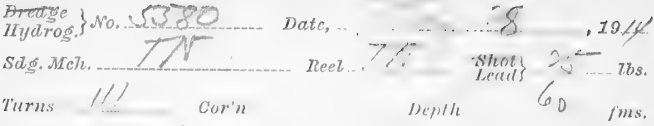

Turns /I! Cor'n Amt, Bot'm

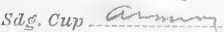
Sample...

Character of Hollom Cis. $\because$.

Thermom. $\left\{\begin{array}{l}\text { Afulies } \\ \text { Nos. }\end{array}\right.$ Vor'n $\left\{\begin{array}{l}\text { Instr. } \\ \text { stem. }\end{array}\right.$

Bottom Reading

Cor'dl. Trmp).

$\operatorname{remp} . \operatorname{surf}\left\{\begin{aligned} \text { Time } \\ \text { Time }\end{aligned}\right.$

Density: Surf.

Wind: Direction.

Buromet. $=$ is $4(6)$

Weather

62 fins.<smiles>C1C[C@H]2CC[C@H]12</smiles>
For't

Direction and Foree $\left\{\begin{array}{l}\text { Sur/ace } \\ \text { of Current. }\end{array}\right.$

Iride'..

Generat Locality

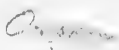

rosition Heceta Light lucers $17^{\circ} \mathrm{E}$ (true) distant

Chart used: No, Goro Ku'n...

Drifl: Direction

Distance.

Depth of Haul

Apparatus and Rig used

Remarles: 
$11-1023$

SOUNDING IVIRE.

, M. DOWn. UT. AI.

FMN.

PREDGING CABLE.

a 157 i

101)

200

30()

400

500

(i) 0

T00

800

?)(

1000

1100

1200

1is)

1400

1500

1(io)

1700

1800

1900

2000

2100

2200

$2: 300$

$\because 100$

2) 00

$\because 600$

2500

$\because 400$

s?)(

$: 1000$

\$100

3200

i3ison

3100

3) 500

3000

:300

:300

:भ९)

.1000

I1)

1200

4800

4100

1500

1600

1700

4800

4900 


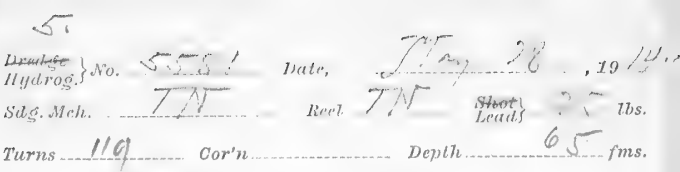

Sag. Cup _... Altming Amt
Samplite

Character of

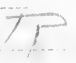

Thermom. $\left\{\begin{array}{l}\text { Make } \\ \text { Nio. }\end{array}\right.$

Cor'n $\left\{\begin{array}{l}\text { Instr. } \\ \text { Stem. }\end{array}\right.$

Botlom Rearlins

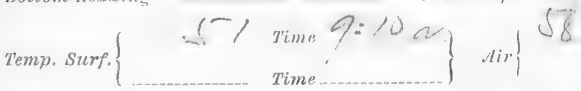

Inensity: surf.

Wint: Jirestion

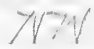

fims.

?o. Ame of

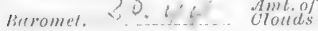
Foref

Wealher is e

Direction and Force
of Current. $\left\{\begin{array}{l}\text { Surfase } \\ \text { Jottom }\end{array}\right.$

Tide

Aeneral Locality

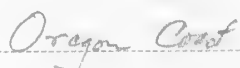

rositionffeceta Leght hears $N 76^{\circ} \mathrm{E}$ (true) distant chert used: No, G.OOD Inl'n.

mift: Direstion

Dislanes

Depth of Haul

Apparatus and Rig used

Remerks: 
SOIJNDING WIRE.

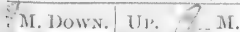

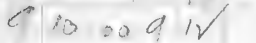

FMS.

DREDGIN $*$ CATBLI:

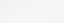

M. Dows. ITr. II.

100

200

300

400

500

(i)

700

800

900

1000

1100

1200

1800

1400

1500

1600

1700

1800

1900

2000

2100

2200

2300

2400

2500

2000

2700

2800

2900

3000

3100

3200

3300

3400

3500

$\$ 3600$

3700

3800

8900

4000

4100

4200

4300

4400

4500

4600

4700

4800

4900 


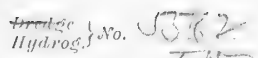

Sils. Meh. Reel

$\int^{2} a 28$

, 1914 $7 /$ strat $24-7 b s$

Turns 1/2 Cor'n

Sag. Cup

Character of Bollom

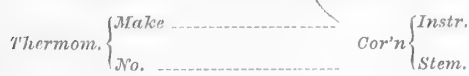

Bottom Reading Amt. Bot'm

Temp. Surf. $\{$ Time

Density: Surf. fins.

Wind: Direction.

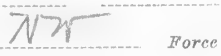

Baromet? ? Yv Amt of

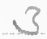

(

State of

Weathrr

$\therefore$

Direction and Forec
of Ourrent. $\left\{\begin{array}{l}\text { Surface } \\ \text { liollom, }\end{array}\right.$

Tide

General Lorality

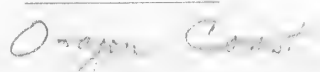

$C^{2}$

Position Heceta Leglet hears $174 / 2^{\circ}$ E(true) Listant chart used: wo. 6000 $\pi d^{\prime} u$

Hrift: Disprlion

Jistamer

Depth of Haut

Apparatus and Kis used.

Time towing

7 . $m$.

Remurhs: 
SOUNDING WIRE

$\because$ Mr. DOWN. Tr. TI. $\therefore 9 \mid 2010092230$
FIIS.

DREDGING ("AIBLE

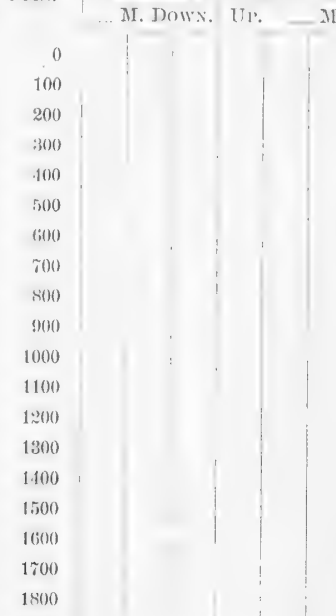
M.

1900

2000

2100

2200

2300

$\therefore 100$

$\because 500$

$\because 600$

2พิ)

2500

2900

3000

$\$ 3100$

3200

3300

3.100

3.500

$\$ 3600$

33600

$: 800$

3900

1000

1100

4.200

1:30()

1400

1500

1600

.1700

1800

f 1900 


\section{7}
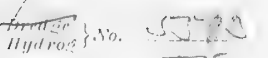

SHC...Heh.

Hate,

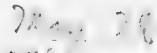

$, 70)$

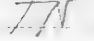

knd

thots

$\therefore$ lis.

Turns II/7)

Cor'n

Depth

Co 4 fis.

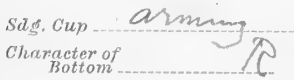

Ant Bot'm
Samople

Thermom. $\left\{\begin{array}{l}\text { Make } \\ \text { Nio, }\end{array} \quad\right.$ Cor'n $\left\{_{\text {sism. }}^{\text {Instr. }}\right.$

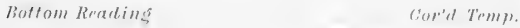

Temp. Surf. $\left.\left\{\begin{array}{l}\text { Time } \\ \text { Time }\end{array}\right\} \operatorname{Air}\right\}$

llensily: Sirr/.

Wind: Dîrection

linemmet. ?o. to

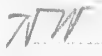

fims

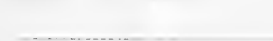

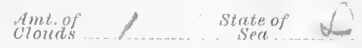

Direction and Force $\left\{\begin{array}{l}\text { Sitwerm } \\ \text { of Current. }\end{array}\right.$

rid.

Limurel Lorelil!

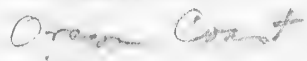

Position Heceta Light heass $M 73^{\circ} \mathrm{F}$ (true) diatant

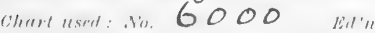

Hrift: Mirestion

Jistenture

Denth of Haul

Apparatus and tis used.

limmerke:

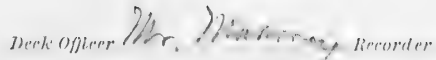


SOUNINNG TVIIRE.

$\therefore$ M. 1) W. (TH. SM. 92029
FNS.

DREDGING CAIBLF.

| ... M. DeWx. UI. ... M.

100)

200

$: 300$

.100

500

(i)o

ז00

sol

(9)0

1000

1100

1200

1300

1.100

1500

16(6)

1700

$1 \times(9)$

1900

2000

2100

2200

21300

2100

2)

2600

2700

$\because 800$

2000

3000

3100

$: 1200$

$: 300$

3400

3500

$: 2400$

3500

3500

3900

.1000

4100

4200

4300

4.100

.500

4000

1700

1800

f(90) 
8

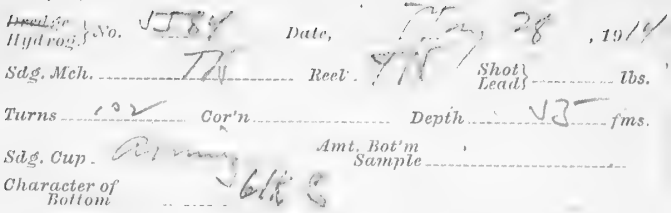

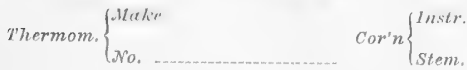

Bottom Reading Cor'd Temp.

Trill. Surf. \{ Tim, ?:??

Time

lensit!l: surf:

Wind: Direction

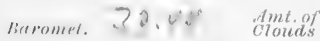
State of

II"Lather ing

Direction and Force
of Current. $\left\{\begin{array}{l}\text { Sulfur } \\ \text { Bottom }\end{array}\right.$

Title

(innerul horatily

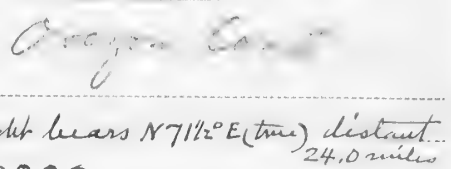

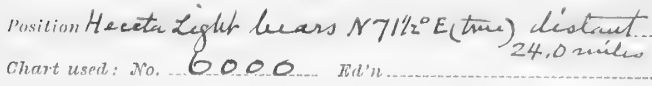

1) rift: Direction Distance

1)epth of Haul

Apparatus and Ri@ used

Time tow in:

h. $m$.

liemertis:

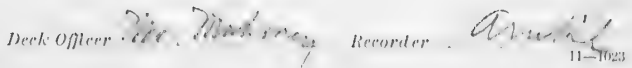




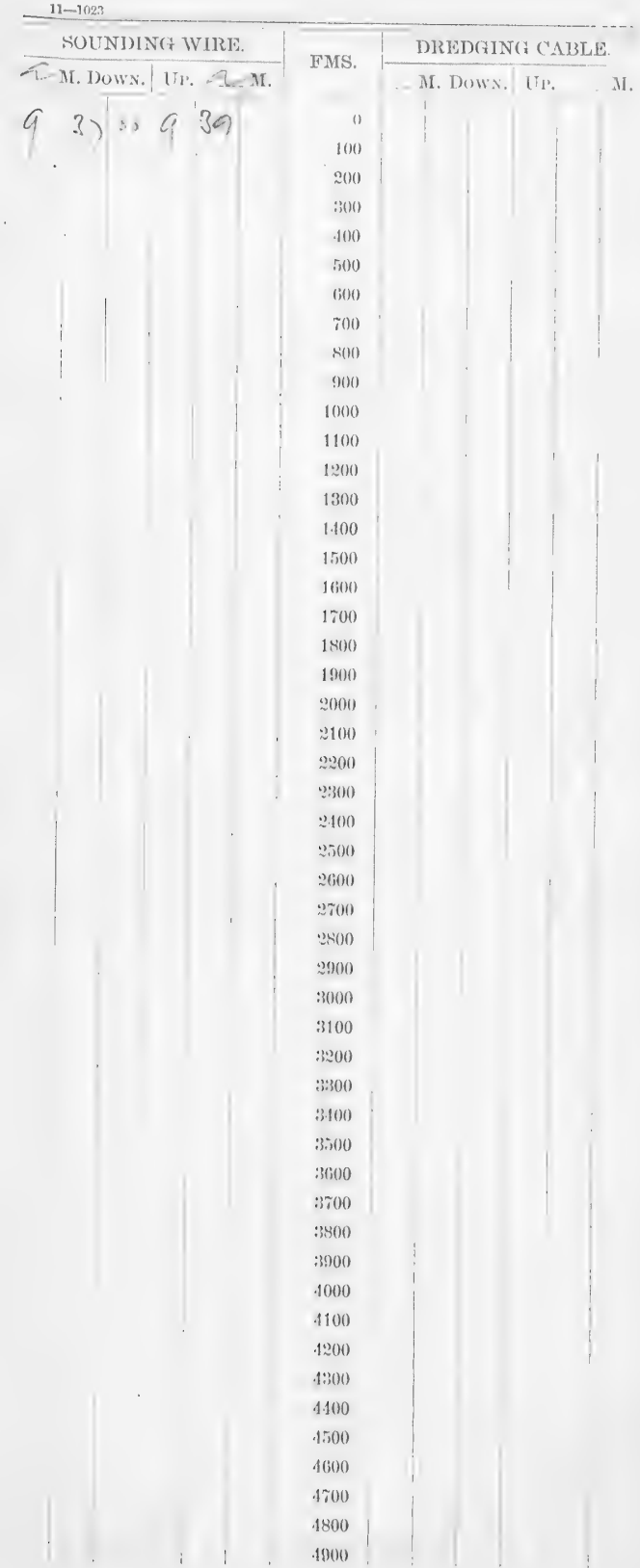


SOUNIING WIIRE.

Tux. Down. UP. \&-M. 2
FMS.

DIREDGING C CABLI:.

M. Down. U1. _... M.

1

100

200

$\because 00$

$+100$

500

(60)

700

$\mathrm{s}(0)$

(9)()

1000

1100

$1: 00$

$1 \%(0)$

1400

1500

1600

1700

1800

1900

2000

2100

2000

s3:

2400

2500

2600

2800

2स00

2900

:3000)

$\$ 3100$

3200

:31300

3.100

3.500

$: 3600$

37\%0

$\$ 3800$

$\$ 3900$

4000

4100

1200

$4 \div 00$

$\$ 100$

1500)

fitor)

1700

1800

f!)(0)
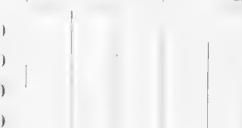

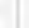

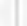


10

mydros tor is Silg. Mch.

Rerl.

Turns

102

cior'n

Depth

seads - 'W - tbs.

sas. Cup

Character of

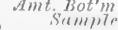
$\sqrt{6}$ fons.

Thermom. $\left\{\begin{array}{l}\text { Mrater } \\ \text { No. }\end{array}\right.$

Cornn $\left\{\begin{array}{l}\text { Instr. } \\ \text { Stem. }\end{array}\right.$

Bottom Reading Cor'd Temp.

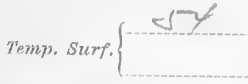

Time

Time

Jensity: surf.

Wind: Direction

Brormet. ?20.

Weather

2

1) irection and Force $\begin{aligned} & \text { Surfues } \\ & \text { of Current. }\end{aligned}$

Tide

lieneral Louretily

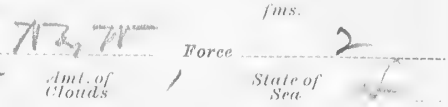

$\operatorname{Air}\{\sqrt{6}$

0.22 .4

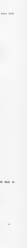


211-110.1

SOLNIDING IVIRE.

I M. IOW. UT. .... M.

$1002.10^{\prime} 01$

FMS.

DREDGING CABLE.

…... M. Down. UP. M.

1)

100

2011)

$: 300$

400

500

(j)0

700

800

()(1)

1000

1100

$1: 00$

1300

1400

1501)

(1)ion

$1 \% 00$

1800

1900

2000

$\because 100$

2900

$2: 300$

200

$\therefore 500$

9600

2 2 200

2800

2900

3000

3100

$: 300$

$: 3: 00$

3.100

3500

3600

:ะช00

: 500

$\$ 900$

4000

4100

4300

$4: 00$

4400

4500

$4(6) 0$

4700

4800

(19)0 


\section{1}

I) real in

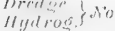

siats. Meth.

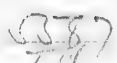

I) al",

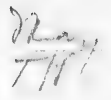

38

Sitom , $20 \%$

tos.

Turns log Cor'n

Depth

19

fims.

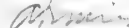

Amt. Bot'm

Sdg. Cup

Character of Bottom

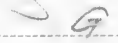

Thermom. $\left\{\begin{array}{l}\text { Make ............. } \\ \text { Non. }\end{array}\right.$ Cor' $\left\{\begin{array}{l}\text { Instr. } \\ \text { Stem. }\end{array}\right.$

Bollom Readins

Cor'te Termp.

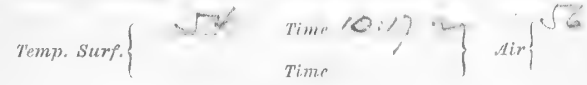

Density: Surf.

fims.

Wincl: Direstion

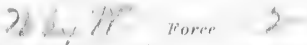

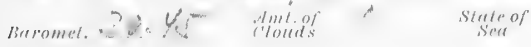

Hnallurr Qu.e.

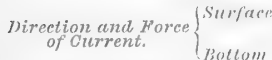

Tirle

General Locality

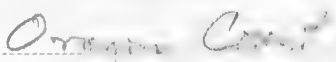

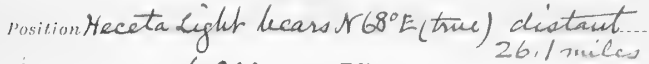

chart uspl: No. 6000 Ed'n

Drift: Direction

Distance

Depth of Haut

Apnaratus and Ris used

Remitrles:

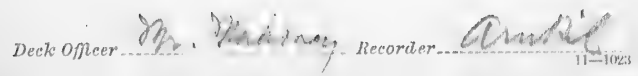


Cim. Down. $1077301020 \%$
FMS.

DREDGING ( A ABLE

M. Dow. UT. . . .

()

101)

200

:301

100

ก(1)

(i)

700

(4)0

900

1000

1100

1200

1800

1400

1500

1600

1700

1800

1900

2000

3100

2200

2300

2400

2500

2000

2700

2800

2900

3000

8100

3200

$\$ 3300$

3400

3500

3600

3700

3800

3900

4000

4100

4200

48300

4400

4500

4000

4700

4800

4900 
SOUNDING WIRE.

? ML. JuNN. ITI. ? JL. $3|2 y| 00 / 0 \mid 0 / 0$.
FMS.

DIRTAING (GABLA:

.... M. Down. I†'. _. M.

100

$201)$

:3(4)

1111)

500

(i) 00

500

800

(100)

1000

1100

1200

1300

1.100

1500

$16(0)$

1700

1:(1)0

1900

2000

$\because 100$

2000

$2: 300$

$2-100$

2500

2000

ขัต()

3900

:?)ा

:3010)

$\$ 3100$

:3: $)(\}$

:in: 100

::10)

Biivol)

:aivo)

:iт(1)

:34010

:3!)

$.1(1006)$

1100

. 1300

f:30)

4100

liso0

I 1600

.1700

18(1)

4900 
13

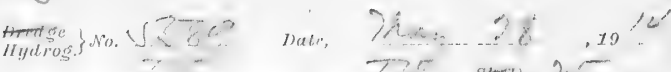

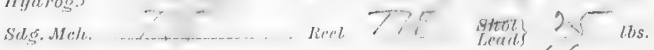

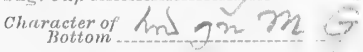

Thermom. $\left\{\begin{array}{l}\text { Mule } \\ \text { No. }\end{array}\right.$

$$
\text { Corn }\left\{\begin{array}{l}
\text { instr. } \\
\text { stem. }
\end{array}\right.
$$

Bottom Rereading ('orts Temp).

Temp. Surf $\left\{\begin{array}{c}\text { Time } \\ \text { Time }\end{array}\right.$

Density: Surf. fms.

Wind: Direction

Baronet.

71

Fores

Weather

State of

Direction and Force
of Current.

Tide

General Locality

Orestes Cone

Position Heceta Light hears $165 \%$. E (trine) distant chart used: No. 60.0. Hin 27,8 mile Drift: Direction Distance

Depth of Haul.

Apparatus and Rig used

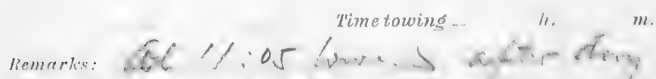

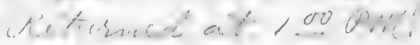

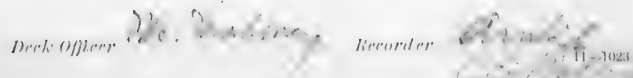


SOUNDING WIRE.

G.r. nowx. It: Gex.

$\therefore l_{0} 0013$ tis

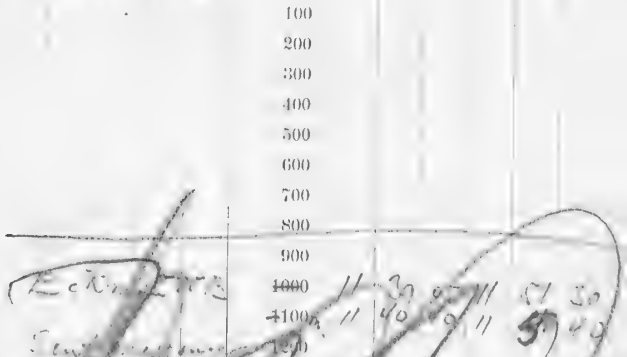

.

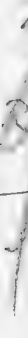

ac

Sic. $81=5,0^{1600}$

is
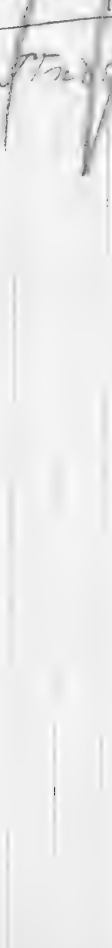

?... sina

atofin

$2: 011$

$23(90)$

2100

2500

2(1)

2ร00

ख्यक्ष

:

:30u(x)

$\$ 1(10)$

:ख)(0)

:3:it)

3400

3500

$: 3000$

3700

3800

3900

4000

4100

4200

4800

4400

4500

4600

4700

4800

4900 


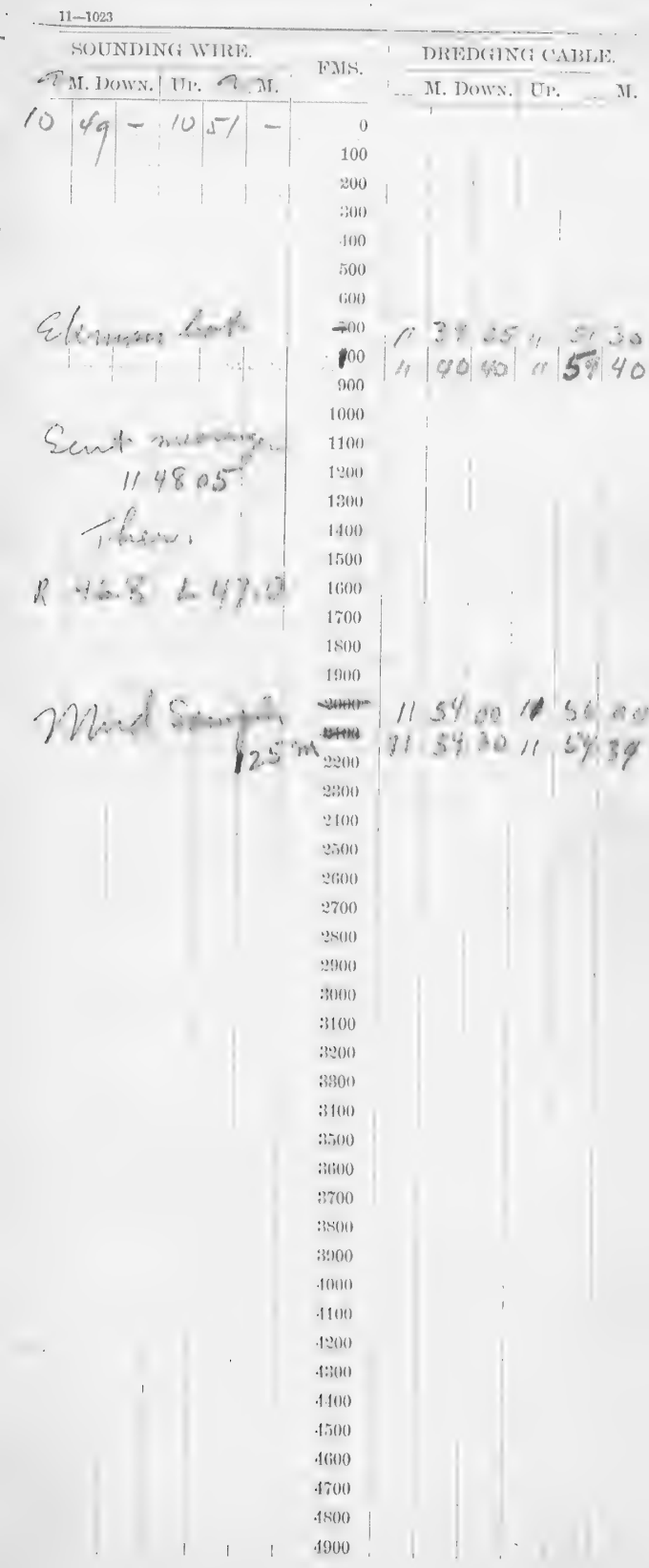




\section{5}

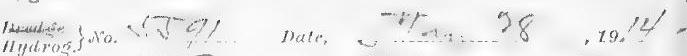

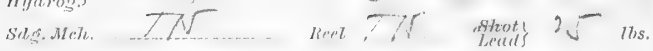

Turns 145 Cor'n............ Depth...

Sag. Cup ..CLaman-... Amt. Bot'm

character of Bottom

Whermom, $\left\{\begin{array}{l}\text { Haker } \\ \text { So. }\end{array}\right.$

Cor'n $\left\{\begin{array}{l}\text { Instr. } \\ \text { sitem. }\end{array}\right.$

Botiom. Reating

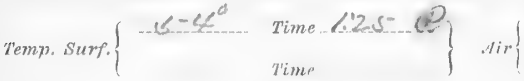
Cor'd Trmp.

Density: Surf. fins.

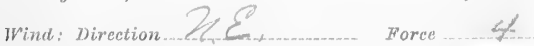

Baromet. 30.38 Amt. of Citids_. State of

Weatien .....LT

Direction and Force
of Current. $\left\{\begin{array}{l}\text { Surfice } \\ \text { Dintom. }\end{array}\right.$

Tide

Generat Lorrelily

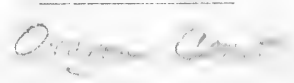

posilion. Hecata Light hears M64 ${ }^{\circ} \mathrm{E}$ (trine) diatant Oherert useet: No 6000 $\pi l^{\prime} n$

7)rift: Direction.

Distance

Depth of Haul

Apparatus and Rig used

Time towing

h.

$m$.

Remurks: :

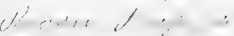

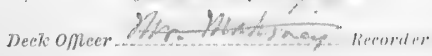

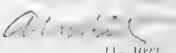



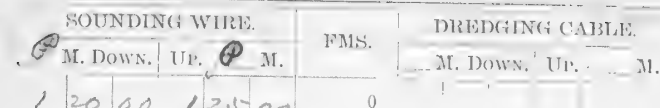

$120 \mid 00.25700 .00$

2010

:30)

140

500

600

(0)

$\times(100$

()o)

1000

1100

12114)

13)

1400

1500

1600

1700

1800

1900

:2000

2100

2200

(29)(0)

2) (0)

:50)

$\because(b)$

2700

2s(i)

:

2000

3100

$\therefore:(1)$

:3:300

:31191)

:3)

abloo

ถึก)

:BS(0)

:300

four)

1100

. $13(x)$

.13(3)

(1) (1)

firot

fritule

1.70)

1500

4000 


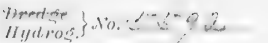

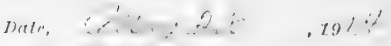

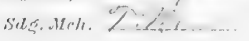

Sinel

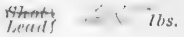

Turns 244

Cor'n

Depth $\angle 3 ?$

fims.

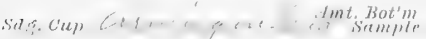

Character of

Bottom

Thermom, $\left\{\begin{array}{l}\text { Make } \\ \text { No. }\end{array}\right.$

Cor'n $\left\{\begin{array}{l}\text { instr. } \\ \text { sirmu. }\end{array}\right.$

Rotlom Renulins

Cor'd Trmp.

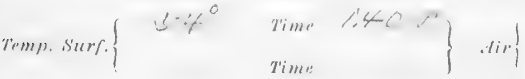

Jensity: Surf.

fins.

Wind: Direction 2,3

Force of

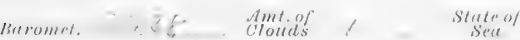

Weather... Lete.

Direction and Force
of Gurrent. $\left\{\begin{array}{l}\text { Surfuco } \\ \text { lioltum }\end{array}\right.$

Tile.

Gennal Lorafily

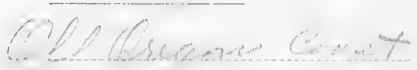

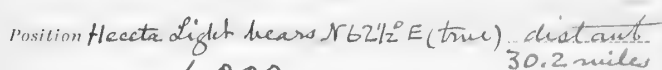
chart used: No...6000 Hd'n

Inifl: Divelion

Disturre

Depth of Haul

Apparatus and Rig used

liemurk: 

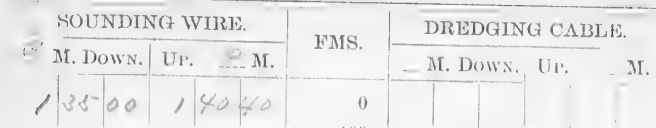

100)

200

:3)

100

500

(5) 0

\%०0

800

()00

(1)0 0

1100

1200

1300

1400

1500

16(00)

$1 \% 00$

1800

1000

2000

2100

2200

$2: 300$

$\because 100$

2500

2600

2700

2800

2900

3000

3100

3200

33300

3.100

3500

3600

$: 3500$

$\$ 3800$

$\$ 900$

.1000

.1100

4200

4:300

4.400

4500

4600

.1700

1800

.900 


\section{i)}

Itretion

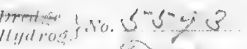

Sta. $.11 \% h$.

a

7) ate,

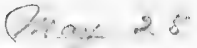

, $19 \cdot \therefore$

Rert.

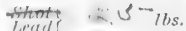

Turns.241 Cor'n

Depth 133 fris.

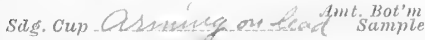

Character of
Bottom

Thermom. $\left\{\begin{array}{l}\text { Matee } \\ \text { No. }\end{array}\right.$

Cor'n $\left\{\begin{array}{l}\text { Instr. } \\ \text { Siem. }\end{array}\right.$

Bottom Reading Cor'd T'emp.

Temp. Surf. $\left\{\begin{array}{ll}s-\psi^{0} & \text { Time lis }-40 \\ & \text { Tim. }\end{array}\right\}$ lir

fins.

Density: Surf.

Force ... 4

Wind: Direction.

Baromet. $30,36 \quad$ Ant.of

State of

lipealher

Direction and Horce $\left\{\begin{array}{l}\text { Sur/ace } \\ \text { of Current. } \\ \text { Bottom }\end{array}\right.$

Tide

limenal boratily

rosition Heceta Light henss $N 64^{\circ}$ E (twue) distant Chart userl: No 6000 Kat's

Jrift: Hirention

Distantere

Depth of Hart

Apparatus and Rie used

limmorte: 


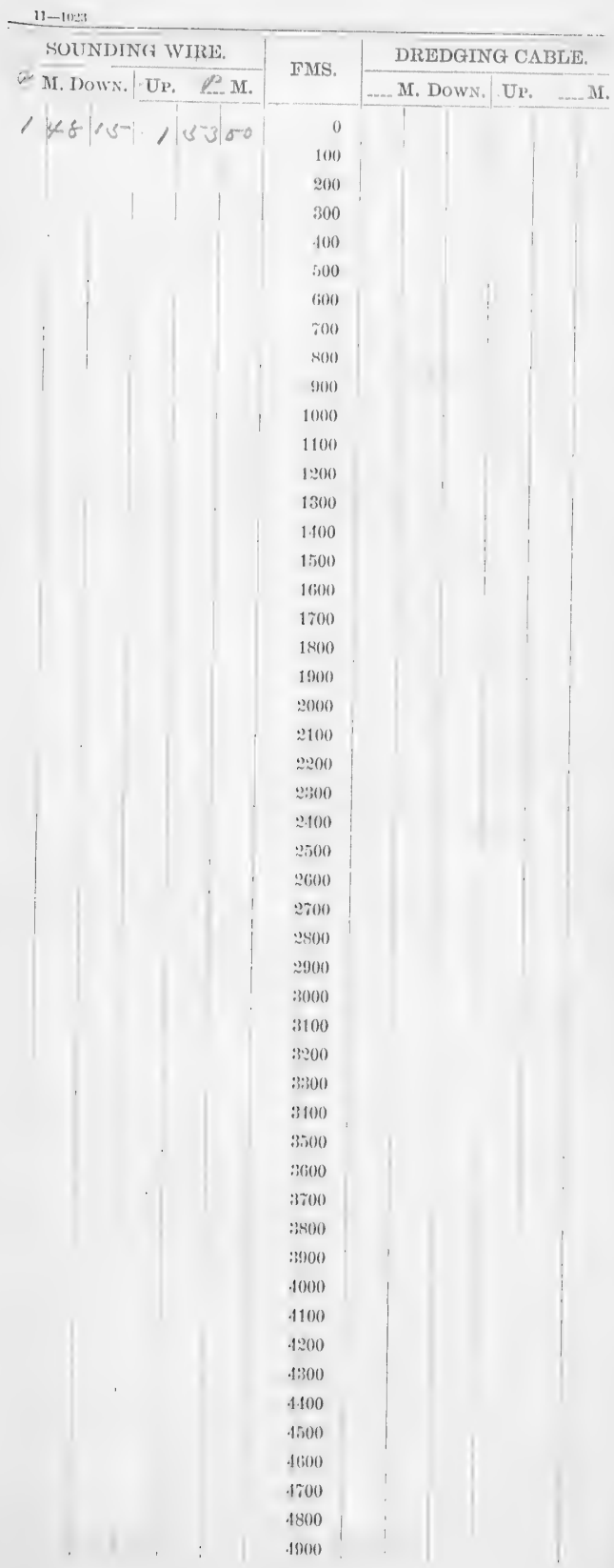


SOUNDING WIRE.

M. Down. Up. M. $\therefore \quad \because \ldots \mid$ an 2 lyige

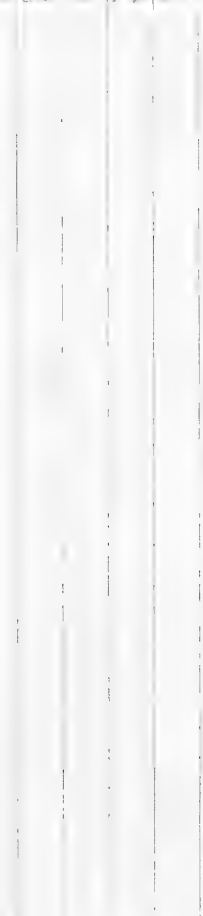

FMS.

DREDGING CABLE.

Q M. Down. Ur. PM.

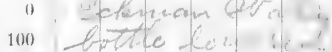

290-

\$90

400 .

500

(i)0

700

800

900

1000

1100

1200

1300

1400

1500

1600

1700

1800

1900

2000

2100

2200

2300

$: 400$

2500

2600

2700

2800

2900

$\$ 3000$

3100

$\$ 3200$

3300

$\$ 400$

3500

3600

$: 3700$

3800

3000

4000

4100

4200

4300

4400

4500

4600

4700

4800

1900
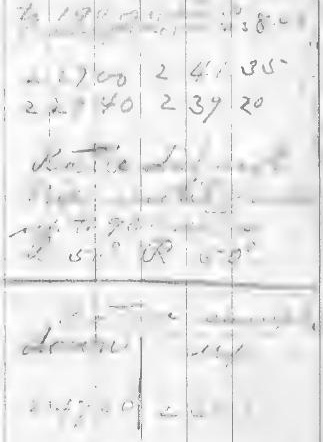


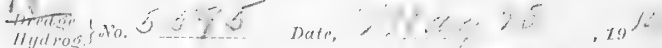
Sdg. Mel, Y Y $\Lambda^{\prime}$

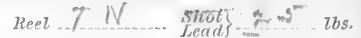
Turns 211_... Cor'n. Depth $1: 15$ fims.

Sils. Cup $:$ Amt. Bot'm

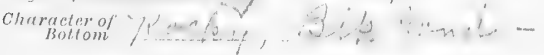

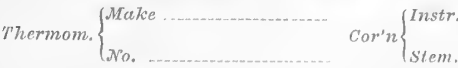

Botlom Rerdins

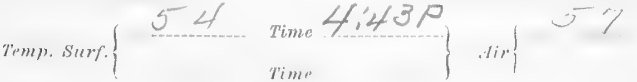

Density: Surf.

fmis.

Wina: Direction. 2) 77,72 Roret

baromet. 30.34 . Ant of 4 state of $L C$

Weathor

$\leftarrow c$

Direction and Foree
of Current.

Tide

(ieneral tocatily - i

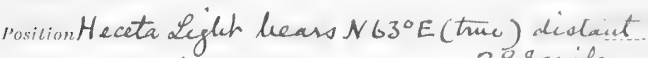
chat userl: No. G.OOO Eil'n

Drift: Direction.

Dislance

Depth of Haut

Apnaratus and Rig used

limmirk's: 


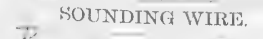

7 .r. Down. $\mid$ UI, $\%$ M. $-13-2425$
FMIS.

DREDGINA CABLE.

... M. Dow. Ur. ... M.

100

200

:301

100

500

(i)

r00

800

100

1000

1100

1:200

1300

1400

1500

$1600)$

1700

1800

1900

2000

2100

2200

$2: 30()$

$\because 400$

4500

2600

2700

2800

2900

3000

3100

3:00

3:300

3.100

3500

$\$ 3600$

3700

$\$ 800$

$\$ 3900$

4000

4100

.1200

4:300

4.100

4500

4600

4700

4800

4900 


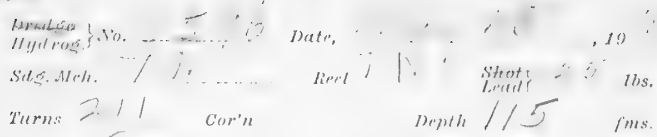

Bottom Reading Cor'd Temp. Temp. Surf. $\left\{\begin{array}{ll}\text { Time } 0,00 \% \\ \text { Time. }\end{array}\right\} \operatorname{Air}\{$ Itensity: Surf:

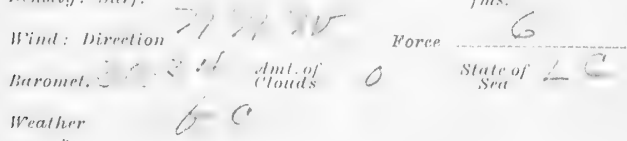

Depth of Haul

Apparatus and Ris used 
$\rightarrow$ SOUNIDINA WIRE.

MI, Down. UI'. .... MT.

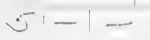

FMS.

DREDGING CAIBTW.

100

200

$: 300$

400

500

(i00)

rou

800

(9)1

1000

1100

1200

1300

1400

1500

1600

1700

1800

1900

2000

2100

2200

$3: 300$

$\because 100$

2500

$\because 600$

2700

2800

2000

3000

3100

3200

$3: 00$

3400

3.500

$: 300$

3 :

3800

3900

1000

4100

4200

4800

4400

4500

4600

1700

4800

4900

M. I)ows. II.
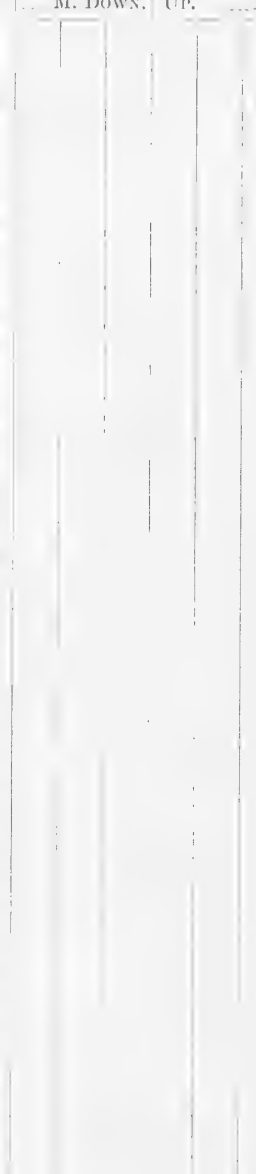
... MI. 


\section{vl}

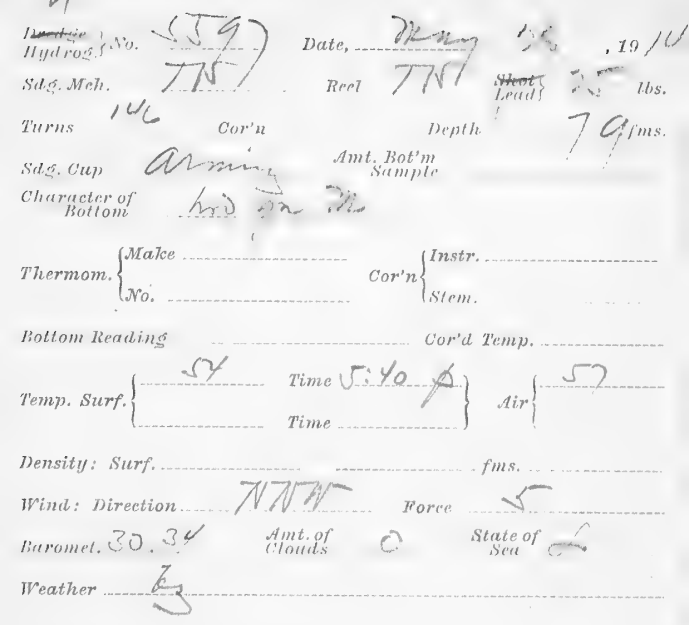

Direction and Foree
of Current. $\left\{\begin{array}{l}\text { Surfaes } \\ \text { Bottom }\end{array}\right.$

Tille

Gimeral Loralily

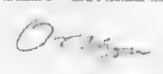

,

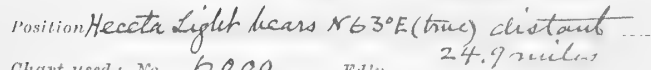

chart used: No, ...6000 $F d^{\prime} n$

Drift: Mirection

Dislamer

Depth of Haul

Apparatus and Rig used

lirmurtis: 
SOUNDING WIIRE. M. DOWN. Ur. $\odot$ M. DREDGING CAISLE.

$\sqrt{40}-\sqrt{4} 4=$ IMS.

M. I)OW. UP. I.

\% 0 ()

800

900)

1000

1100

$1: 00$

1800

1400

1500

1600)

1700

1800

1900

2000

2100

2200

2300

2400

: $250(1)$

2600

$2 \%(0)$

$2 \times 006$

2904

$: 3000$

3100

3200

:3:30

3104

$\therefore 00$

360

3400

:3800

$\$ 9000$

$1000)$

1100

1300

1.300

4.100

4500

s600

1700

1800)

4900 


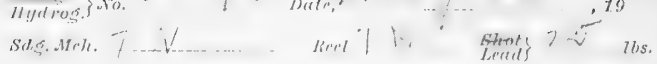

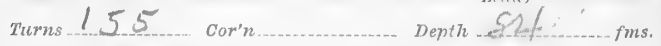

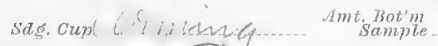

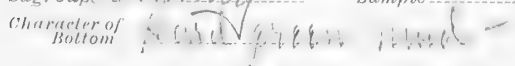
Whermom. $\left\{\begin{array}{l}\text { Mratie } \\ \text { lor. }\end{array} \quad\right.$ Corn $\left\{\begin{array}{l}\text { Instr. } \\ \text { Stem. }\end{array}\right.$

Bottom Reading Cor'd Temp.

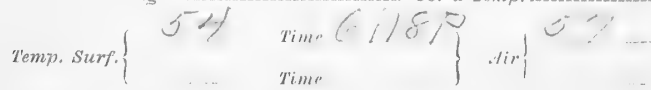
1)ensily: Surf. IMint: lirection 2,3120 fins.

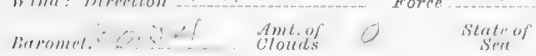
Weather 27

Direction and Force
of Ourrent.

Tide.

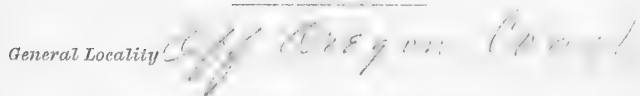
"osition Heceta Light hears $163^{\circ} \mathrm{E}$ (thue) dieturt Chart used: No. 6000 Ed'n

Drift: Direction. Distance. Depth of Haut

Apparatus and Rió used

Remurls: 
(10)11

1000

1100

1200

1900

1400

1500

1600

1700

1500

19(10)

2000

2100

2000

2300

:1010

2500

$\because 600)$

2 200

34र(1)

2)(14)

:30)

$\$ 100$

3200

:1300

$\$ 3100$

3.100

:3610)

:1700

:490)

:?100)

.1000

1100

.1200

$1: 300$

f. 100

f500

(bio)

1700

4800

4000 
Silo $M \cdot t_{1}$

Nhot

Turns 172 Cor'n

Depth fms.

Sids. Cup

Amt. Bot'm
Sidminle

character of Bottom

Thermom. $\left\{\begin{array}{l}\text { Alake } \\ x_{0} .\end{array}\right.$

Cor'n $\left\{\begin{array}{l}\text { Instr. } \\ \text { stem. }\end{array}\right.$

Bottom Reading

Temp. surf.

Cor'd Temip.

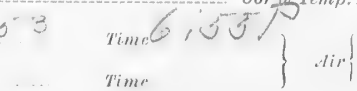

Density: Sitrs.

fins.

Wina: Direction 27.

Baromel. ? 5 Amt.of

Force 6

Wealizer . G C

State of 15

Direction and Force
of Current. $\left\{\begin{array}{l}\text { Surface } \\ \text { Bottom }\end{array}\right.$

Tille

General horalily

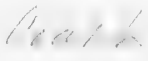

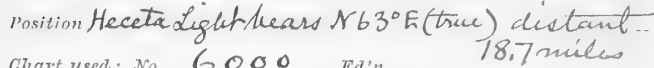
chart used: No Gooo

Drift: Direction

Distance

Depth of Haut

Apparatus and Rig used

Remurks: 
11-1023

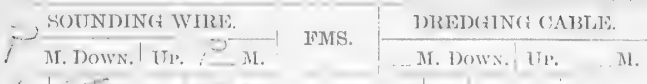

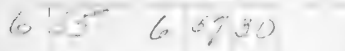

(1)0

200

:30)(

i(ii)

;00

(i)

io()

80

(1)(0)

$10(0)$

150()

$1: 00$

120)(

1.100

1500

1600

1700

$1+00$

1906)

2000

2100

2200

2:30)

$\because 100$

250()

:(10)

皮()

ㅂiv()

$\because 3(0)$

$: 3000$

$\$ 100$

:ivor)

:3:30)

:310)

i3)

:itiou

:3(v)

:3)(x)

:39)(9)

1000

fl $(x)$

1200

4:30)

1100

Aino)

.1600

.1700

1900)

490() 


\section{$;,$}

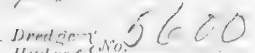

IIydion f lio.

Sreg. Aet. - i it

lietr,

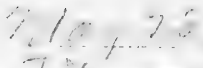

, $10 / 6^{\circ}$

Turns fi 7 Cor'n

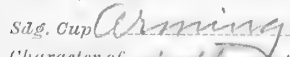

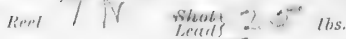
neptie

fins.

Amt. B3ot'm

Hotrom
Hot

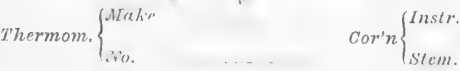

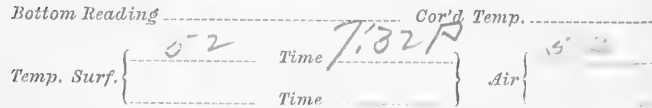

l)ensity: Surf.

fins.

Wind: Direction...27 Foree

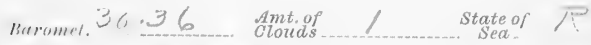

Walher of 1,2

Direction and Force $\left\{\begin{array}{l}\text { Surfuce } \\ \text { of Current. }\end{array}\right.$

Tills.

Generel Lorktity

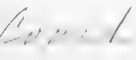

Position Heceta Light hears $N 63^{\circ} \mathrm{E}$ (truc) distant

chart used: No, 6000 Fit'n

Drift: Direetion

Distance

Dept72 of Haut

Apparatus and Rig used

Remartes: 


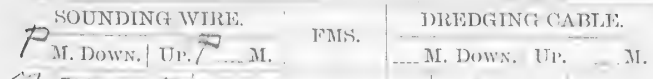
$732-736-$

300

.400)

500

(i)lo

700

Sरा0

๑)(0)

1000

1100

120()

130()

1400

1500

1000

1700

1800

1900

$?(1) 0$

2100

$2 ! 04)$

$2: 3000$

$31(0)$

2500

1)(ii)()

घ7()

प्रक(3)

:9)(

:3000

$\because 100$

$: 2000$

$3: 100$

:10)

:3:50

: 2400

:300

:3800

:3000

$.100(1)$

.1106

$1 \div()()$

4:30)

1100

1ivo()

dito()

1700

1800

1900 


\section{6}

I) renil

IIylrons sto.?

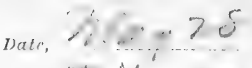

18

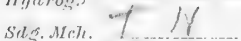

serel $N$

Shotits is the

Turns 150 cor'n

1) (2)

fims.

siles.

Cherracterof Bottom

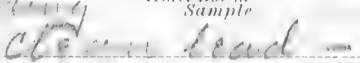

Thermom, $\left\{\begin{array}{l}\text { Hake } \\ \text { fru. }\end{array}\right.$ Cor'n $\left\{\begin{array}{l}\text { Instr. } \\ \text { Stem. }\end{array}\right.$

Bollom kisutins

Temp. Surf. \{

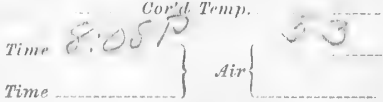

Time

fins.

7)(usity: surf.

Wind: Direction

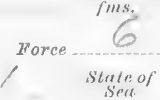

baromet. $30.3\left(0 . . \quad \begin{array}{l}\text { Amt of } \\ \text { clouds }\end{array}\right.$

Seret.

Weattier

i)

Direction and Force
of Current. $\left\{\begin{array}{l}\text { Surfues } \\ \text { lottom }\end{array}\right.$

tides...

General Lorrtity

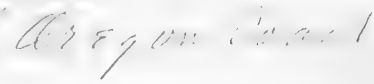

Position. Heceta Leght hears $163^{\circ}$ E (twac) distant wherl usert: vis. 6000 kit'n

Drift: Dimetion

Histanee

Depth of Haut

Apparatus and Rigused

Remarks: 
SOUNDING IVIRE.

M. mown.| TH. ... MT. c) 08
FMS.

-... M. Jow. Th. -M.

$10(3)$

200

300

1110

500

(i) (i)

100

Si( )

(1)()

1000

1100

120()

1300

1.100

1500

1600

1700

1800

1900

2000

2100

2क्य)

2):

$\because 100$

2500

seithe

witi)

$\because 400$

(3)

:300)

:3100

:3)

:洨保)

:110

init)

:3(ic)0

:300

:

:19)()

. $10(1)$

.1100

.1200

.13000

111)0

tis(t)

litio)

17i(1)

4 ario

4000 


\section{$\gamma$}

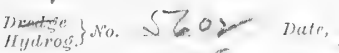

Sas. Mch. 7.15

lieel

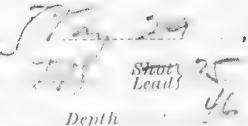

, $12: \div$

Turns ... Cor'n

sas. cup LQ t-zamaus

Amt, Bot'm

Character of

Botlom

Thermom. $\left\{\begin{array}{l}\text { Malce } \\ \text { No. }\end{array}\right.$

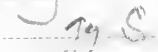
Sample.

Bottom Reading

Temp, Surf. \{

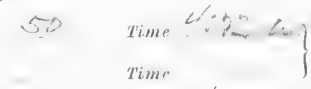

Cow'd Temp.

D)ensity: surf:

Mind: Direction

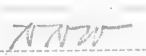

Force

Buromet. 20,2, Amt of

Cor'n $\left\{\begin{array}{l}\text { Instr. } \\ \text { Stem. }\end{array}\right.$

Weather Zow,

Direction and Forec $\left\{\begin{array}{l}\text { Surface } \\ \text { of Current. }\end{array}\right.$

Iidi

General Locality

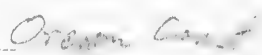

posilion Heceta Lighh hears $\$ 74 \%: \mathrm{E}$ (Tme) distant chart used: No 6000 Ed'n

Drift: Direction

Distance

Jepth of llaut

Apparatus and Ris used.

Remarks: 
SOUNIDNO

(MI. DOWN: UTI. A... MT.

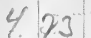

WMS.

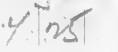

DREDGIN( ; CABT,E.

MI. Dow: II. M.

0

100)

200

:30k)

.100

500

(i0)

700

s(i) 0

900

1000

1100

1200

1800

1100

1500

i(i)()

1700

1800

1900)

:21)(10)

2100

2000

2:300

2100

:5000

(2)(0)

2\%00

3500

300

: :00(1)

:3100

mon

:300

3100

(3)ivo

$\therefore(60)$

:3700

2800

3900

.1000

.1100

. 200

4300

1100

.1500

. 6000

1700

1800

. (ต)० 


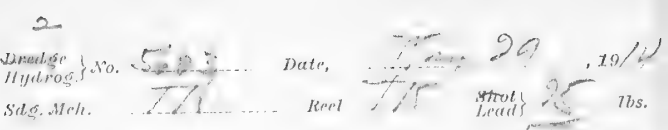

Turns 105 Cor'n

DeptTh fins.

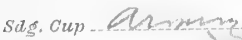

character of Botlom Amt. Bot'm

Thermom. $\left\{\begin{array}{l}\text { Make ..... } \operatorname{Cor}^{*} n\left\{\begin{array}{l}\text { Instr: } \\ \text { No. }\end{array} \text { Stem. }\right.\end{array}\right.$

Bottom Reading

Cor'd Termp.

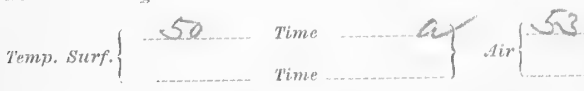

7)ensity: Surf.

Wind: Direction.....

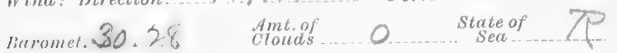

Weather $\ldots$

Direction and Force
of Current. $\left\{\begin{array}{l}\text { Surfact } \\ \text { Bottom }\end{array}\right.$

Tide

General lotatily

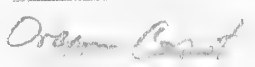

Position Heceta Light bears $\$ 85^{\circ} \mathrm{E}$ (thue) distant...

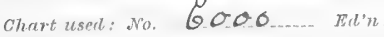

Drift: Direction

Distance

Depth of Haut

Apparatus and Ris used

lipmortis:

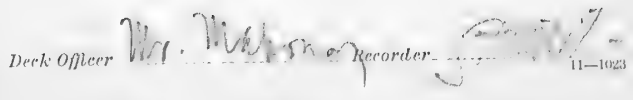




\section{- SOUNDING WIRE.}

- $\mathrm{M}$, Down. UP. $\sqrt{ } \mathrm{M}$. $v(\pi)-502-$
FMS.

DREDGING CABLE.

I

.... M. Down.| UP. _... M.

()

100

200

300

I(K)

;i)0

(i) ()

\%ol

SOO

!)()

1000

1100

12(1)

$13(0)$

1.400

1500

1600

$1 \% 00$

$1 \mathrm{~s} 00$

1900

2000

2100

200

$2: 300$

$\because 900$

प:

") $(\theta 0)$

2700

$2 \mathrm{~s}(0)$

2900

$3(000)$

3100

:3200

:3:3(1)

:3(19)

3iकo()

:360)

:ละ)

$: 3800$

$: 1900$

(1)(0)

1100

(12)0

4:300

.1100

firo0)

(t) 600

1700

1800

4900

M. 


\section{3.}

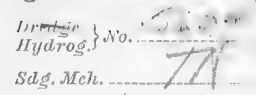

Sag. Mch.

Turns
Inete, +2

l)eplth

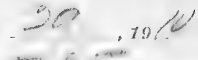

shiol lbs. 63 tims.

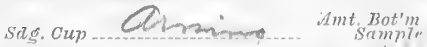

Character of Bottom. $\sim$ cleatin

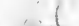

Thermom. $\left\{\begin{array}{l}\text { Make } \\ \text { No. }\end{array}\right.$ Cor'n $\left\{\begin{array}{l}\text { Instr. } \\ \text { Strm. }\end{array}\right.$

Bottom lreading Cor'd Temp.

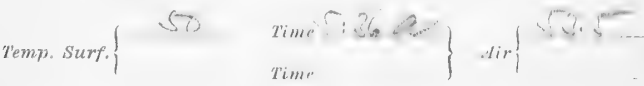
Hensit!l: sur\%.

"Hinl: Hirertion

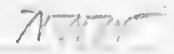

fims.

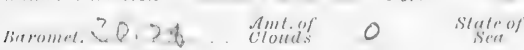

wealter. Zt.

Direction and F"orce
of Ourrent. $\left\{\begin{array}{l}\text { Surface } \\ \text { Bistlom }\end{array}\right.$

Tille

General tocality

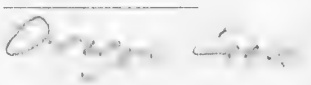

rusilion Heceta Light hears E (time) hidat. Chert used: . Wh. 6000 Haln

Drift: Direction.

Distance

Depth of Haut

Apparatus and lich used.

Remurhs: 
11-10:3

SOUNDING WIRE.

$\therefore$ MI. Down. U1. II. $5.56-58$
DREDGTNG CABLE

_... M. Down. UP. … M.

(1)

10)

200

:300

400

.50

(i)

700

4i)(

()())

1000

1100

1200

1300

1400

1500

[(60)

1700

1800

1! (m)

2000

2100

2900)

2:301)

$\because 100$

280

$2060)$

2 \%

2900

:9)(1)

:30)0

3100

:3:(0)

:3is(y)

$: ; 100$

$330(0)$

:B(ti)

:ล7(6)

:34)(1)

:3!)(0)

11906)

.1100)

1.206

9:301)

1.100

tivon

1400)

4700

1800

1900 
Hectares to lo.

Silk. Al sh.

Turns
Tine -

$130 \ldots . . \operatorname{cor}^{\prime} n$

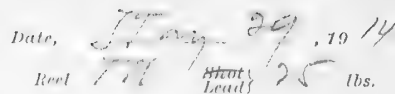

Depth.... 20 fins. Sag. Cup arming Ant. Bot'm Character of
Bottom set on 72

Thermos. $\left\{\begin{array}{l}\text { Muir } \\ \text { No. }\end{array}\right.$

$\operatorname{Cor} n\left\{\begin{array}{l}\text { Instr. } \\ \text { Stem. }\end{array}\right.$

Bottom Reading Cor'd Temp.

Tromp. surf: \{ Time $6: 14 x$ Time

Density: Surf. fins.

Wind: Direction

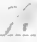

Baronet, $30.28 \quad$ Amt. of Fire

Weather

$7_{i=1}$

Direction and Force
of Current. $\left\{\begin{array}{l}\text { Surface } \\ \text { belem, }\end{array}\right.$

Tile?

General Locality

O, O.

Cant

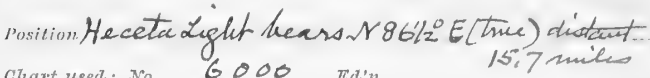
chart used: No. Go 00 Tin

Drift: Direction Distance.

Depth of Hart

Apparatus and Rig used

Time touring

h.

$m$.

Remrertis:

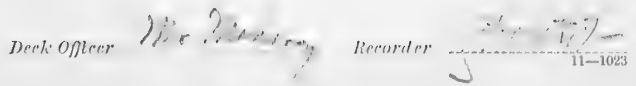




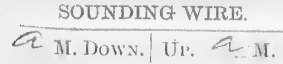

FMS.

$6|14|-6|16|-$

DREDGING CABLE.

II.

00

200

8300

100

500

(i) $(1)$

\%(0)

800

:400

1000

1100

12(14)

1300

1400

1500

1600

1700

1800

1900

2000

2100

2000

25300

2900

250)

20100)

2ซา11

2S(0)

25)

:3)(1)

$\$ 3100$

:300

:3i300

:300)

(3)iा)(

:3600

:7800

3400

:34)(1)

100)

1100)

4200

1300

4.400

tis 00

f(i)o

400

1800

1900 
11-1023

$\Leftrightarrow \mathrm{M}$. Down. Ur.

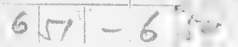

FMS.

I(r)

2(1)

; $3(1)$

.100

i) (1) ()

(i)

(5)( )

S(1)

900

1000

1100

1200

1300

1400

1500

1600

1700

1800

1000

2000

2100

2200

2300)

2400

2500

2600

2700

2800

2900

$\$ 3000$

3100

3200

3300

3400

3500

$\$ 3600$

3700

3800

3900

4000

4100

4200

4300

4400

4500

4600

4700

1800

4900

DRIDDGING CABLF.

_._. Down. UP. _ II.

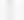
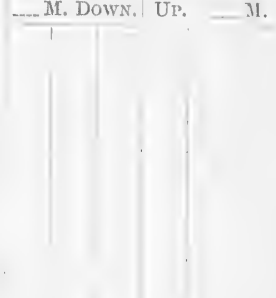

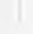




\section{6.}

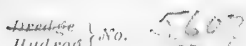
Mydios, sio. Meh.

Dute,

71,25

$19 ! 1$

Turus $>2-C_{-}{ }^{\prime} n$

Depth

68

lbs.

sas. Cup _fl-po2im Amt. Bot'm
Sample

Character of fin 9 .

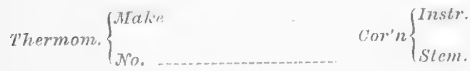

Bottom Reading

Cor'd Temp.

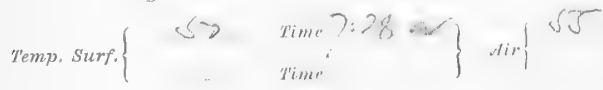

Density: Surf. fms.

Wind: Direction.... DTRP

Buromel. $30.38 \ldots .$. Amt of

0 Stateof

Weather

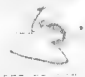

Direction and Force
of Current. $\left\{\begin{array}{l}\text { Surface } \\ \text { Bottom. }\end{array}\right.$

Tille

General Loeselity
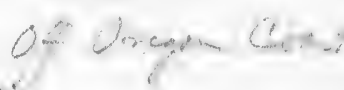

vosilion Heceta Ligh hears $182 \% 2^{\circ}$ E (truc) olistant.. chart used: No...

Drift: Direction

Distance

Depth of IIaut

Apparatus and Rig used

Nemurlis: 
SOUNDING WIRE. FM. Hown. U1. F. M. $7.80-73030$
FNS.

DREDGING OABLIE.

M. Down. UT.

M.

1600

1700

18100

1900

$:(9) 0$

$\because 100$

$\because 200$

:2:31)

2300

2500

$2(6) 0$

2700

$28(0)$

$\therefore 900$

$\therefore(000$

3100

$\because 200$

:3:300

3100

3.500

:अ(i)

$: 3700$

8900

:300

1000

4100

f(20)

. $1: 000$

4100

4500

$4(0) 0$

4700

$4 \times 0$

t!xol

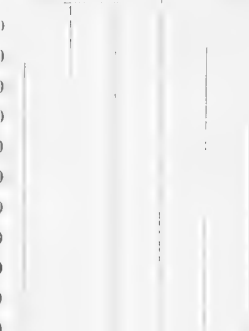


1900

$\because 000$

2100

2000

2:300)

$\because 100$

3500

2600

2700

खsं

a900)

$: 1000$

\$100

:1900

:300

$\$ 100$

3500

:1600

$3 \%(0)$

$: \mathbb{4} 4(30$

3900

4000

.1100

4200

A:Bo(

.1100

1500

.1000

1700

4800

.900 


\section{8}

sills. .lleht

$+5$

Cor'n

Turns

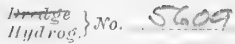

$2 i$

Date,

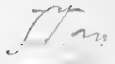

2.4

limel

Shot
leftil?

l) $m$ inth

fins.

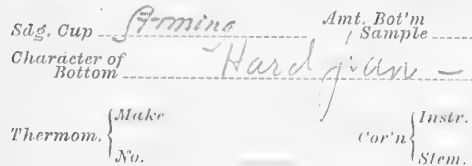

Bottom Reading Cor'd Temp.

Temp. Surf. $\left\{\begin{array}{ll}\ldots \text { Time } & \text { Time }\end{array}\right\}$ Air

Density: Surf.

fins.

Wind: Direction...72:21: 2

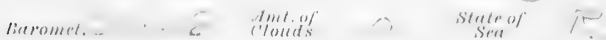

Herefluer

Direction and Force
of Gurrent. $\left\{\begin{array}{l}\text { Suriam } \\ \text { Bottom }\end{array}\right.$

Titl.

(imenerat boerality

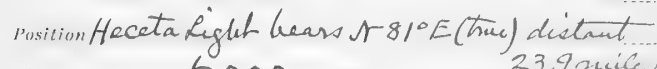
chart uspel: No. 6000 Iidn....

Drifl: Direction.

Distance

Depth of Haut

Apparatus and Ris usel.

limmorks: 
$: 3200$

:moc

3100

3500

: :ivo

$: 5 \% 00$

3800

3900

1000

1100

1200

. 1300

1.100

tivol)

4600

1700

4800 


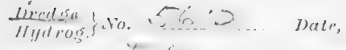
site. $11 \% \%$.
Y 1,
Iinel
$\because M$
Yhot
, 10.
Turns I! ! . Cor'n
heplh : :-
7 bs.

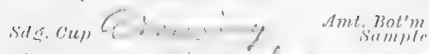

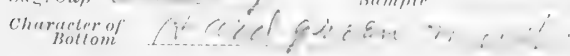

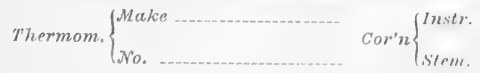

Bottom Reading

Cor'd Temp.

Temp. Surf. $\left\{\begin{array}{ll}\text { Time } 0,1-1 \\ \text { Time }\end{array}\right\}$ Air

Density: Surf.

fins.

Irind: Himertion

Horre

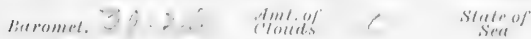

Itwether

Direction and Force
of Current. $\left\{\begin{array}{l}\text { Surface } \\ \text { liottom }\end{array}\right.$

Tilil"

Generat Tocality
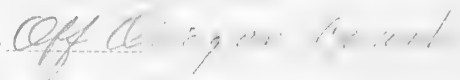

rosilion Heceta Light bears $r 80 \% \%^{\circ}$ E(true distast chart used: No. 6000 Ed'n 24,8 nute

Drift: Direction.

Distance...

Depth of Haul

Apparatus and Rig used.

limmurk: 


\section{SOUNDING WIRE.} A M. Down. Up. A... M. $8 / 3|-8 / 16| 0$

100

200

300

400

500

600

700

800

900

1000

1100

1200

1800

1400

1500

1600

1700

1800

1900

2000

$2100^{\circ}$

2200

2300

2400

2500

2600

2700

2800

2900

3000

8100

3200

3300

3400

3500

3600

3700

8800

8900

4000

4100

4200

4300

4400

4500

4600

4700

4800

4900

DREDGING CABLE.

_....M. Down. UP. _... M.

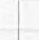

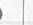

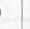

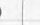

.

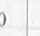


10

Dredge
Hydras No. $56 / 1$ Date, $6 / 2 \%, 19$

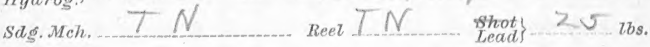
Turns 125 Corn Depth $6 \%$ fins.

Sag. Cup Amt. Bot'm

Character of
Bottom

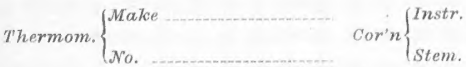

Bottom Reading Cor'd Temp.

Temp, Surf. $\{$ si Time $8: 5-0$

Time

$$
\operatorname{Air}\{-6
$$

Density: Surf. fms.

Wind: Direction Q7.6\%, 2\%. Force 6 Baronet. $30,2 \&$ Amt of State of

Weather

Direction and Force
of Current. $\left\{\begin{array}{l}\text { Surface } \\ \text { Bottom }\end{array}\right.$

Tide

General Locality Off Orgour each

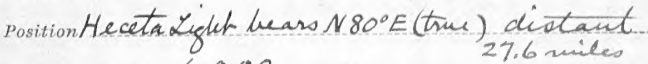
Chart used: No. 6000 Finn

Drift: Direction Distance

Depth of Haul

Apparatus and Rig used

Time towing h. $m$.

Remarks:

Deck offer

Recorder 
SOUNDING WIRE.

A M. Down. UP.A M. $88-0 \leqslant 0$ \& 5230
FMS.

DREDGING CABLE.

0

….M. Down, UP. _......

100

200

300

400

500

600

700

800

900

1000

1100

1200

1800

1400

1500

1600

1700

1800

1900

2000

2100

2200

2300

2400

2500

2600

2700

2800

2900

3000

3100

3200

3800

3400

3500

3600

3700

3800

3900

4000

4100

4200

4300

4400

4500

4600

4700

4800

4900

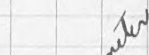

j. $A 1^{4}$

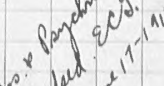

$e^{3}$ 
56 fons. -

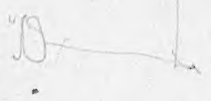

Heape $\angle Q M$ crazo of unat will oil

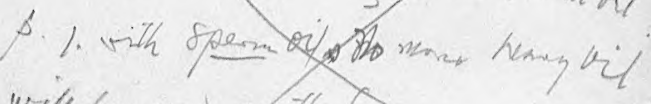
will be used of thly mor an

irien stwoes.

$$
\text { Ba dinatin of } c 0
$$

